HRI-RECAPP-2009-014

\title{
Non-universal scalar mass scenario with Higgs funnel region of SUSY dark matter: a signal-based analysis for the Large Hadron Collider
}

\author{
Subhaditya Bhattacharya $^{a 1}$, Utpal Chattopadhyay ${ }^{b 2}$, Debajyoti Choudhury ${ }^{c 3}$, \\ Debottam Das $^{b 4}$ and Biswarup Mukhopadhyaya ${ }^{a 5}$ \\ (a) Regional Centre for Accelerator-based Particle Physics, \\ Harish-Chandra Research Institute, \\ Chhatnag Road, Jhusi, Allahabad 211 019, India. \\ (b) Department of Theoretical Physics, Indian Association for the Cultivation of Science, \\ 2A 8 2B Raja S.C. Mullick Road, Kolkata 700 032, India. \\ ${ }^{(c)}$ Department of Physics and Astrophysics, University of Delhi, Delhi 110 00\%, India.
}

\begin{abstract}
We perform a multilepton channel analysis in the context of the Large Hadron Collider (LHC) for Wilkinson Microwave Anisotropy probe (WMAP) compatible points in a model with non-universal scalar masses, which admits a Higgs funnel region of supersymmetry dark matter even for a small $\tan \beta$. In addition to two and threelepton final states, four-lepton events, too, are shown to be useful for this purpose. We also compare the collider signatures in similar channels for WMAP compatible points in the minimal supergravity (mSUGRA) framework with similar gluino masses. Some definite features of such non-universal scenario emerge from the analysis.
\end{abstract}

\footnotetext{
${ }^{1}$ subha@hri.res.in

2 tpuc@iacs.res.in

${ }^{3}$ debchou@physics.du.ac.in

${ }^{4}$ tpdd@iacs.res.in

${ }^{5}$ biswarup@mri.ernet.in
} 


\section{Introduction}

Low energy Supersymmetry (SUSY) [1] is a strong candidate for physics beyond the Standard Model. A general framework like the Minimal Supersymmetric Standard Model (MSSM) $[1,2]$, however, suffers from a large number of a priori unrelated parameters. This lack of predictability can be minimized only if one assumes a definite mechanism for breaking SUSY. The minimal supergravity (mSUGRA) [3] model that assumes gravity to be the mediator between the hidden sector wherein supersymmetry breaks and the observable sector (where the MSSM exists) is relatively economical in this respect. Starting from only a few parameters, renormalization group evolutions (RGE) and the use of radiative electroweak symmetry breaking (REWSB) generate all the MSSM parameters at the electroweak scale.

The mSUGRA scenario has a remarkable simplicity of principle, an economy of parameters and features that at least partially ameliorate potentially disastrous consequences in low energy physics. From a more agnostic standpoint, however, there is no strong reason to restrict ourselves to such universal models. For one, even with gravity conveying supersymmetry breaking, the soft SUSY-breaking terms need not be universal at the supergravity scale, but would depend on the structure of the Kähler potential. Similarly, large nonuniversal corrections may accrue to the soft parameters as a result of the evolution between the Planck scale and the gauge-coupling unification scale $\left(M_{G} \simeq 2 \times 10^{16} \mathrm{GeV}\right)$ [4]. These and other related issues have led to several studies of non-universal scalar [5-15] and gaugino mass $[16,17]$ models. Non-universal scalar masses may appear due to a non-flat Kähler metric [18], or, for example, from $S O(10) D$-terms [12-14]. However, any such nonuniversality, at the electroweak scale, would lead to low-energy flavor changing neutral current (FCNC) processes (through SUSY loops) [19]. The existing data on flavor physics thus impose severe constraints on any nonuniversality in scalar masses, in particular for the first two families. The restrictions on the third generation scalars (and the Higgses) from FCNC data are not too severe though.

It turns out that both FCNC and CP-violation constraints may be best tackled if one assumes the first two generations of scalars to be multi-TeV and (quasi-)degenerate in masses $[20]^{1}$. Clearly, allowing universal scalar masses at the gauge coupling unification scale would not satisfy the above objective because either $(i)$ the REWSB constraint would prohibit such large scalar masses for a reasonable set of values of the gluino masses, or $(i i)$

\footnotetext{
${ }^{1}$ We remind the reader that satisfying constraints imposed by electric dipole moments of electron and neutron would require very large scalar masses if we like to have finite values for the CP-violating SUSY phases.
} 
one must have very large gaugino masses, so as to allow very large scalar masses, thereby worsening the fine tuning problem [21]. We recall that, within the MSSM, the naturalness problem and its solution revolve around the third family, as well as the gaugino and Higgs scalar mass parameters. As long as the third generation scalars and the electroweak gauginos are on the lighter side, any quantitative measure of naturalness would stay within an acceptable domain. Furthermore, constraints from FCNC and CP-violation are relatively weak in such a scenario with an inverted mass hierarchy $[22,25]$.

In this work, we consider a particular non-universal scalar mass scenario (NUSM), namely that of Ref. [5]. The model addresses the FCNC issue by invoking very large masses for the first two generations of squarks and sleptons. As is well-known, such a solution is difficult to achieve within the mSUGRA scenario as the requirement of REWSB prevents the scalar masses from being too large. In the present context, this is circumvented by allowing the third generation squark masses and the Higgs scalar mass parameters to be small. This very smallness also serves to keep the degree of fine-tuning within control.

As far as the third generation sleptons are concerned, a very small SUSY-breaking mass at the GUT scale is not phenomenologically viable since the larger Yukawa coupling serves to drive down the mass of the lighter stau, thereby rendering it the lightest of the supersymmetric partners (LSP) at the electroweak scale. Consequently, the SUSY-breaking mass in this sector has to be sizable ${ }^{2}$. Rather than introducing a new parameter, we shall assume it to be same as that of first two generations of squarks or sleptons. To summarize, at the GUT scale, all sfermion masses are diagonal; and, apart from those pertaining to the stop and the sbottom, are universal. The last-mentioned, along with the Higgs scalars, have a vanishing mass at this scale. While this construction might seem artificial, note that this accords a special status to only those fields that are expected to play a direct role in EWSB. Interestingly, the model satisfies the WMAP constraint [26] on neutralino relic density for a large region of the parameter space without requiring any delicate mixing of Binos and Higgsinos. For simplicity, we confine ourselves to a universal gaugino mass and a vanishing trilinear soft-breaking parameter $\left(A_{0}\right)$ at $M_{G}$.

We investigate how such a scenario can leave its fingerprint on numbers measured at the Large Hadron Collider (LHC). Such fingerprints are of value if ways can be devised to distinguish this scenario from an mSUGRA one with, say, similar gluino masses. For

\footnotetext{
${ }^{2}$ However, in analyses with Higgs-exempt no-scale SUSY model [23] or in a model with gaugino mediation [24] one may avoid such charged LSPs at the electro-weak scale by using non-zero Higgs scalar masses at the unification scale. In these scenarios the no scale boundary conditions are also valid for sleptons.
} 
this, one has to perform a multichannel analysis $[15,17,27,28]$ studying several final states simultaneously.

A promising signal of supersymmetry (with a conserved R-parity) comprises large missing transverse energy, accompanied by jets and leptons with varying multiplicities. An analysis in different channels, compared with that of a similar mSUGRA scenario may lead to a significant hint of the non-universality. In the present analysis, we assess the accessibility of our non-universal scalar mass model (NUSM) at the LHC. We find that the direct pair production of stops and sbottoms as well as their cascading down from gluino decays lead to the possibility of four-lepton final states as a distinct signature of this scenario. Additionally, we also analyse the two-lepton and the three-lepton final states. This includes opposite sign dilepton, same-sign dilepton and trilepton final states. All these analyses are done also for mSUGRA so that the multipronged approach of analysing for different channels may become more conclusive.

The paper is organized as follows. In Section 2 we describe the NUSM model, apply cosmological constraints on neutralino dark matter and use low energy constraints such as those from $b \rightarrow s+\gamma$ or $B_{s} \rightarrow \mu^{+} \mu^{-}$. We also identify benchmark points for our analyses of collider signals at the LHC. In Section 3, we pinpoint our strategies for collider simulations and report the numerical results. Finally, in Section 4, we summarize our results and conclude.

\section{The Non-Universal Scalar Mass model (NUSM) and benchmark points}

\subsection{The NUSM parameter space}

The NUSM model [5], at the scale $M_{G}$, is characterized by five parameters, namely,

$$
\tan \beta, m_{1 / 2}, m_{0}, A_{0} \text { and } \operatorname{sign}(\mu)
$$

The parameters, here, play rôles similar to those in mSUGRA except for a subtle and important difference in the scalar sector. Masses of the first two generations of scalars (squarks and sleptons) and the third generation of sleptons are assigned the value $m_{0}$. However, the Higgs scalars and the third family of squarks have vanishing mass values at $M_{G}$. Here, $m_{0}$ is allowed to be up to tens of TeVs. As has already been stated, we limit ourselves to a vanishing $A_{0}$ in this analysis. We have considered $\mu>0$ in this analysis. 
The NUSM admits a smaller pseudoscalar Higgs boson mass $m_{A}$ on account of the Large Slepton Mass (LSM) renormalization group effect [5] for large $m_{0}$. With such a $m_{0}$, the LSM effect causes $m_{H_{D}}^{2}$ to become large and negative and this may happen for even a small $\tan \beta$. This, in turn, reduces the masses of the pseudoscalar Higgs boson $(A)$, the CP-even heavy Higgs boson $(H)$ and the charged Higgs bosons $\left(H^{ \pm}\right)$. In this scenario, $\mu$ is quite insensitive to a change in $m_{0}$ [5], since the Higgs and the third-generation squark masses at $M_{G}$ are free of the latter. In fact, $\mu$ is completely independent of $m_{0}$ up to one-loop, whereas the two-loop contributions to its RGEs result in only a tiny dependence on $m_{0}$. Recall that, in mSUGRA on the contrary, $|\mu|$ decreases significantly with an increase in $m_{0}$. Whereas this led to a very small $|\mu|$ for a large $m_{0}$ in mSUGRA, giving the so called Hyperbolic Branch/Focus point (HB/FP) $[29,30]$ region that is close to the upper limit of $m_{0}$ satisfying REWSB for a given $m_{1 / 2}$, there is no HB/FP type of effect in NUSM and $\mu$ stays reasonably independent of $m_{0}$. It turns out that the lightest neutralino is highly Bino-dominated (with a small Higgsino admixture) throughout virtually the entire parameter space of NUSM. Along with the resonance condition, namely, $2 m_{\tilde{\chi}_{1}^{0}} \simeq m_{A}\left(m_{H}\right)$, the small Higgsino content allows the LSP to have the right degree of pair-annihilation via s-channel Higgs-exchanges, so as to satisfy the WMAP limits on the neutralino relic density. It is important to note that, excepting for LSP-stau coannihilation, the Higgs-pole annihilation mechanism is the only one in NUSM that reduces the relic density from overabundance to an acceptable degree of abundance. Thus, unlike in models such as the mSUGRA, here one does not need any delicate mixing between a Bino and Higgsinos in order to satisfy the WMAP data. Such Higgs-pole annihilations that occur for large $\tan \beta$ in mSUGRA is typically known as the funnel region $[31,32]$. NUSM has an extended funnel region that spans from low to high $\tan \beta$.

\subsection{Cosmological and low energy constraints in NUSM}

Assuming that dark matter was generated thermally, the limits on the cosmological relic density from the WMAP data [26] impose severe constraints on supergravity type of models wherein the lightest neutralino $\widetilde{\chi}_{1}^{0}$ becomes the LSP for most of the parameter space $[33,34]$. We now perform an analogous analysis for the NUSM. For a given set of parameter (vide eq.(1)) values, the supersymmetric particle spectrum is generated using SuSpect v2.34 [35]. This, then, is used as an input to micrOMEGAs [36] for computing the neutralino relic density. 
The recent WMAP data [26] stipulates that, at the $3 \sigma$ level,

$$
0.091<\Omega_{C D M} h^{2}<0.128
$$

where $\Omega_{C D M}$ is the dark matter relic density in units of the critical density and $h=0.71 \pm$ 0.026 is the reduced Hubble constant (namely, in units of $100 \mathrm{~km} \mathrm{~s}^{-1} \mathrm{Mpc}^{-1}$ ).
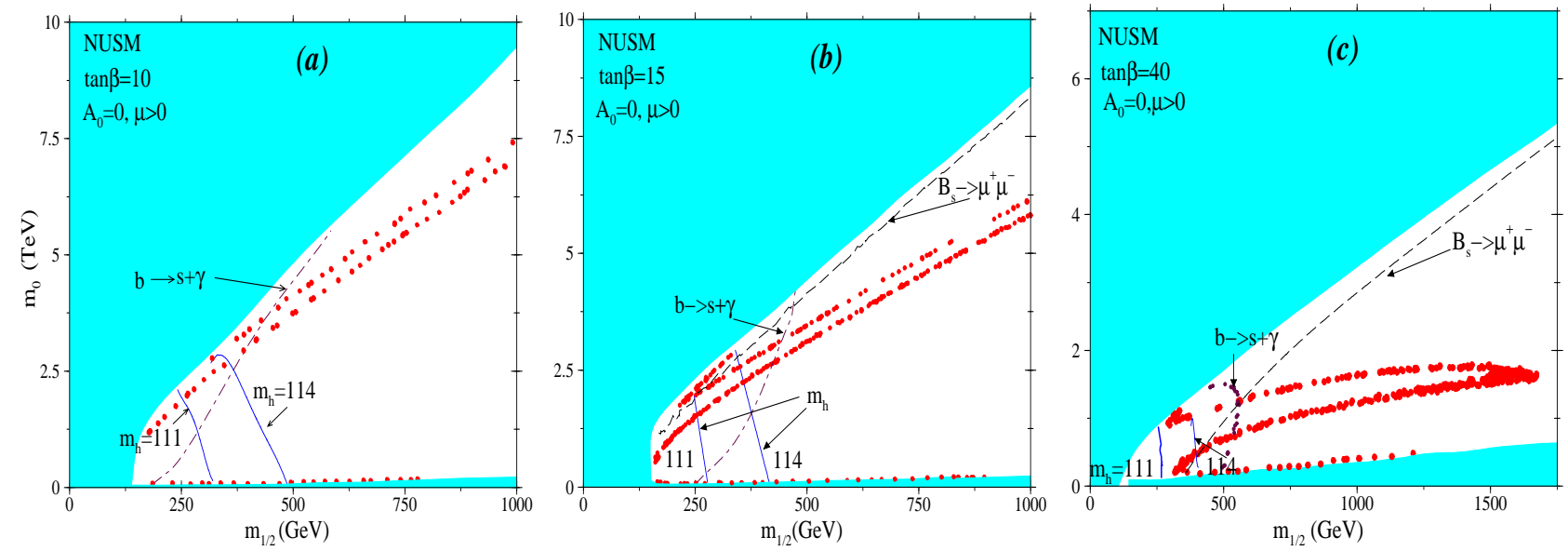

Figure 1: (a) WMAP allowed regions in the $m_{1 / 2}-m_{0}$ plane for $\tan \beta=10$ and $A_{0}=0$ with $\mu>0$ for NUSM are shown in red dots. Lighter Higgs boson mass limits are represented by solid lines. Dot-dashed line refers to $b \rightarrow s \gamma$ limit. The entire region is allowed by $B_{s} \rightarrow \mu^{+} \mu^{-}$data. (b) Same as (a) except that $\tan \beta=15$. The $B_{s} \rightarrow \mu^{+} \mu^{-}$bound is shown as a long-dashed line. This eliminates a small strip of region below the discarded top (cyan) region. (c) Same as (b) except that $\tan \beta=40$.

In Fig.1, we display the allowed regions in the $m_{1 / 2}-m_{0}$ plane for three values of the ratio of the Higgs vacuum expectation values, namely $\tan \beta=10,15$ and 40. The thin (cyan) sliver at the bottom is ruled out as, for such values of the parameters, the lighter stau becomes the LSP. The upper (cyan) region is rejected primarily on account of the failure in the breaking of the electroweak symmetry via radiative means. In other words, for such parameter values, $m_{A}^{2}$ does not acquire a positive value through RG flow. Close to the boundary of this region, several other phenomenological constraints become important. The most important of these pertain to $(i)$ the LEP2 and Tevatron lower bounds for sparticle masses, (ii) sfermions turning tachyonic, or (iii) the appearance of charge and color breaking (CCB) minima. To be allowed, a parameter point must evade all these and other such constraints. Specific details may be found in Ref. [5]. 
Highlighted (in bold — red - dots) in Fig.1 are examples of parameter points that satisfy the WMAP data. There are two distinct regions with acceptable relic density as already mentioned in Sec.2.1. (a) The Higgs pole annihilation region (also known as the funnel region) is characterised by $2 m_{\tilde{\chi}_{1}^{0}} \simeq m_{A}, m_{H}$. In this particular scenario, it extends over the full range of $m_{1 / 2}$ under consideration. The Higgs pole annihilations may occur through $s$-channel pseudoscalar Higgs boson $(A)$ or CP-even neutral $\mathrm{H}$ or h-bosons. NUSM has a bino-dominated LSP similar to what occurs in mSUGRA in its funnel region that satisfies the WMAP data. Similar to the case in mSUGRA, the WMAP satisfied parameter regions of NUSM is also dominantly characterized by the pseudoscalar Higgs boson mediated resonance annihilation. The exact or near-exact resonance regions have very large annihilation cross sections resulting in a high degree of under-abundance of dark matter. The resonance region that satisfies the WMAP data may be a few $\Gamma_{A / H}$ away from exact resonance. The widths $\Gamma_{A / H}$ (of $A / H$ bosons) can be fairly large (e.g. $\Gamma_{A / H} \sim 10-50 \mathrm{GeV}$ ). The WMAP satisfied regions fall on either side of the exact resonance condition thus showing two branches in the figure. (b) The second region, just above the lower ruled-out part, corresponds to the case where the lighter stau is nearly degenerate with the LSP, leading to very efficient LSP-stau coannihilation, thereby reducing the relic abundance to acceptable levels.

Also imposed on Fig.1 are the pertinent low-energy constraints. Whereas non-observance at LEP2 impose a strict bound of $114.4 \mathrm{GeV}$ on the SM Higgs [37], with recent negative results from Tevatron [38] ruling out even somewhat heavier Higgses, the translation of this bound to the MSSM case needs careful consideration. Apart from the parameter-dependence of the cross-sections at LEP2/Tevatron, one needs to account for the uncertainties in computing the mass of light Higgs boson [39], originating primarily from momentum-independent as well as momentum-dependent two-loop corrections, higher loop corrections from the topstop sector etc. Numerically, this amounts to about $3 \mathrm{GeV}$, and we have taken that into account in drawing the solid lines representing this constraint. Additionally, a part of the NUSM parameter space is associated with very light $m_{A}$ even for a small $\tan \beta$ and this may lower the lighter Higgs boson lower bound to a value much smaller than that of the SM Higgs boson limit. We will revert to this while discussing the NUSM benchmark points.

A low energy observable of particular importance is the decay rate for $b \rightarrow s \gamma$ rate $[40,41]$, which, at the $3 \sigma$ level, reads [42]

$$
2.77 \times 10^{-4}<\operatorname{Br}(b \rightarrow s \gamma)<4.33 \times 10^{-4}
$$

We used micrOMEGAs [36] for computation of $b \rightarrow s \gamma$ that in turn refers to Refs. [40,41] for actual computation. Typically, $b \rightarrow s \gamma$ disfavours the small $m_{1 / 2}$ region where the rate is 
below the lower limit. Note, however, that the usual estimation assumes a perfect alignment at high energies between the quark and squark mass matrices. In other words, the (super)Cabibbo-Kobayashi-Maskawa matrix operative for supersymmetric diagrams is assumed to be identical to the usual CKM matrix. However, if one relaxes the above assumption and considers even a moderate amount of $\tilde{b}-\tilde{s}$ mixing at the GUT scale, Eq.3 is no longer an effective constraint for high scale models like mSUGRA. This, on the other hand, will not cause any significant change in the sparticle mass spectra or in the flavor conserving process of neutralino annihilation. We refer the reader to Refs. [43,44] for further discussions on the amount of model-dependence in computing $\operatorname{Br}(b \rightarrow s \gamma)$ in this context.

Since the NUSM scenario may contain a light pseudoscalar Higgs, it is necessary to consider the constraints from $B_{s} \rightarrow \mu^{+} \mu^{-}$. Within the MSSM, the above branching ratio is proportional to $m_{A}^{-4}$ and $\tan ^{6} \beta$ [45]. The recent CDF [46] limit for $\operatorname{Br}\left(B_{s} \rightarrow \mu^{+} \mu^{-}\right)$is given by

$$
\operatorname{Br}\left(B_{s} \rightarrow \mu^{+} \mu^{-}\right)<5.8 \times 10^{-8} .
$$

The branching ratio of $B_{s} \rightarrow \mu^{+} \mu^{-}$is evaluated by using micrOMEGAs [36] that in turn implemented Ref. [47] for the computation. The computation involves inclusion of loop contributions due to chargino, sneutrino, stop and Higgs exchanges. The upper limit of this branching ratio is shown in dashed lines in Fig.1. The white regions above the dashed lines in Figs.1 $(b, c)$ are thus discarded. As mentioned in Ref. [5], the intense coupling region of Higgs bosons that appears when $m_{A}$ is very small is also ruled out in NUSM for the same reason.

\subsection{A few benchmark points}

The NUSM has a large volume of allowed parameter space, especially because REWSB does not prohibit $m_{0}$ from assuming a very large value. We focus here on a few characteristic parameter points that satisfy WMAP as well as low energy constraints. As seen in Figs.1( $a^{-}$ $c$ ), for a given $m_{1 / 2}$, the upper limit on $m_{0}$ decreases with an increase in $\tan \beta$. As an example, for $m_{1 / 2}=1 \mathrm{TeV}, m_{0}$ may well be as large as $7 \mathrm{TeV}$ for $\tan \beta=10,6 \mathrm{TeV}$ for $\tan \beta=15$, and $1.6 \mathrm{TeV}$ for $\tan \beta=40$.

Here, we have preferentially explored those regions in the parameter space which give distinctly different low energy and cosmological signatures as compared to mSUGRA. As we have mentioned before, the Higgs funnel region for mSUGRA is found only for large values of $\tan \beta$. The NUSM is characteristically different from mSUGRA in the sense that funnel 


\begin{tabular}{|c|c|c|c|}
\hline parameter & $\mathrm{A}$ & B & $\mathrm{C}$ \\
\hline $\tan \beta$ & 10 & 15 & 40 \\
\hline$m_{1 / 2}$ & 270 & 255 & 540 \\
\hline$m_{0}$ & 2050 & 2000 & 1250 \\
\hline$A_{0}$ & 0 & 0 & 0 \\
\hline $\operatorname{sign}(\mu)$ & 1 & 1 & 1 \\
\hline$\mu$ & 312 & 291 & 651 \\
\hline$m_{\tilde{g}}$ & 709 & 674 & 1280 \\
\hline$m_{\tilde{u}_{L}}$ & 2100 & 2050 & 1660 \\
\hline$m_{\tilde{t}_{1}}$ & 276 & 248 & 842 \\
\hline$m_{\tilde{t}_{2}}$ & 493 & 465 & 1030 \\
\hline$m_{\tilde{b}_{1}}$ & 390 & 354 & 958 \\
\hline$m_{\tilde{b}_{2}}$ & 434 & 403 & 1020 \\
\hline$m_{\tilde{e}_{L}}$ & 2050 & 2000 & 1300 \\
\hline$m_{\tilde{\tau}_{1}}$ & 2040 & 1970 & 1120 \\
\hline$m_{\tilde{\chi}_{1}^{ \pm}}$ & 196 & 183 & 430 \\
\hline$m_{\tilde{\chi}_{2}^{ \pm}}$ & 347 & 327 & 668 \\
\hline$m_{\widetilde{\chi}_{4}^{0}}$ & 347 & 326 & 668 \\
\hline$m_{\widetilde{\chi}_{3}^{0}}$ & 318 & 297 & 655 \\
\hline$m_{\widetilde{\chi}_{2}^{0}}$ & 197 & 185 & 430 \\
\hline$m_{\widetilde{\chi}_{1}^{0}}$ & 108 & 101 & 226 \\
\hline$m_{A}$ & 259 & 148 & 403 \\
\hline$m_{H^{+}}$ & 272 & 169 & 411 \\
\hline$m_{h}$ & 111 & 111 & 116 \\
\hline$\Omega_{\tilde{\chi}_{1}^{0}} h^{2}$ & 0.105 & 0.102 & 0.130 \\
\hline$B r(b \rightarrow s \gamma)$ & $1.59 \times 10^{-4}$ & $4.65 \times 10^{-5}$ & $2.73 \times 10^{-4}$ \\
\hline $\operatorname{Br}\left(B_{s} \rightarrow \mu^{+} \mu^{-}\right)$ & $4.02 \times 10^{-9}$ & $2.81 \times 10^{-8}$ & $5.29 \times 10^{-8}$ \\
\hline$\Delta a_{\mu}$ & $9.31 \times 10^{-11}$ & $1.59 \times 10^{-10}$ & $6.99 \times 10^{-10}$ \\
\hline
\end{tabular}

Table 1: NUSM Benchmark points A, B and $C$ (masses are in GeVs). The first five parameters define the model, while the rest are predictions.

regions exist even for small $\tan \beta$. Hence, we choose to explore one benchmark point with 
small $\tan \beta$. Additionally, we examine a benchmark point for large $\tan \beta$. The next point to note is that NUSM, typically, has heavier spectra for the first two generations of scalars and the third generation of sleptons. Sfermions become heavier with increase in $m_{1 / 2}$. Therefore, if we like to probe the model in the early phase of the LHC, we would rather select $m_{1 / 2}$ to be relatively small for the benchmark points.

Table 1 lists three benchmark points for NUSM. Point A (for $\tan \beta=10, A_{0}=0, m_{1 / 2}=$ $270 \mathrm{GeV}, m_{0}=2.05 \mathrm{TeV}$ and $\operatorname{sign}(\mu)=1$ ), is associated with reasonably small masses for stop, sbottom, charginos as well as neutralinos. Furthermore, it has a light Higgs spectrum. All these are promising from the viewpoint of early LHC results. Point B of Table 1 refers to a special parameter point $\left(\tan \beta=15, A_{0}=0, m_{1 / 2}=255 \mathrm{GeV}, m_{0}=2.0 \mathrm{TeV}\right.$ and $\operatorname{sign}(\mu)=$ 1 ), for which the Higgs sector is not in the decoupling $[48,49]$ region. Thus, here we obtain a reduced lower limit for $m_{h}$ (close to $\left.M_{Z}\right)$. Point $\mathrm{C}\left(\tan \beta=40, A_{0}=0, m_{1 / 2}=540 \mathrm{GeV}\right.$, $m_{0}=1.25 \mathrm{TeV}$ and $\left.\operatorname{sign}(\mu)=1\right)$ represents a relatively heavier spectrum. However, the relevant parts of NUSM spectra still remain within the LHC reach. We point out that we have relaxed the $b \rightarrow s \gamma$ constraint for points A and B. This is in keeping with the discussion in the paragraph following Eq.3. However, with a small displacement of the parameter point, we would be able to respect the constraint at the cost of having a benchmark point with an upwardly shifted spectrum. Both points A and B obey the constraints from $B_{s} \rightarrow \mu^{+} \mu^{-}$. Point $\mathrm{C}$, on the other hand, satisfies all the constraints, namely, those from $B_{s} \rightarrow \mu^{+} \mu^{-}$and $b \rightarrow s \gamma$, over and above those from the WMAP data.

We also study the collider signatures for mSUGRA scenario at points with the same (or very similar) gluino mass and $\tan \beta$ corresponding to the each of points $\mathrm{A}, \mathrm{B}, \mathrm{C}$. These have been denoted by mSUGRA-A, mSUGRA-B and mSUGRA-C. We must mention that the requirements of obeying the stringent WMAP data as well as the lower bound of the lighter chargino mass did not allow us to choose exactly identical values of the masses of the gluino in each case of the mSUGRA points. This is particularly true for mSUGRA-A and mSUGRA-B that fall in the HB/FP zone. The high scale parameters as well as the low scale soft masses for these points are listed in Table 2, all of them being consistent with the constraint from WMAP. $\operatorname{sign}(\mu)$ is taken to be positive and the trilinear coupling $A_{0}$ is taken to be zero, as mentioned earlier. The corresponding low-energy spectra have also been generated via SuSpect v2.34 using two-loop RGEs. Full one-loop and the dominant two-loop corrections to the Higgs masses are incorporated. We have used the strong coupling $\alpha_{3}\left(M_{Z}\right)^{\overline{M S}}=0.1172$ for this calculation, adopting the default option in SuSpect. We have assumed the top quark mass to be $172.7 \mathrm{GeV}$ throughout the analysis, and no tachyonic 


\begin{tabular}{|c|c|c|c|}
\hline parameter & mSUGRA-A & mSUGRA-B & mSUGRA-C \\
\hline $\tan \beta$ & 10 & 15 & 40 \\
\hline$m_{1 / 2}$ & 253 & 252 & 490 \\
\hline$m_{0}$ & 2740 & 2300 & 2680 \\
\hline$A_{0}$ & 0 & 0 & 0 \\
\hline $\operatorname{sign}(\mu)$ & 1 & 1 & 1 \\
\hline$\mu$ & 139 & 135 & 266 \\
\hline$m_{\tilde{g}}$ & 740 & 725 & 1270 \\
\hline$m_{\tilde{u}_{L}}$ & 2745 & 2320 & 2810 \\
\hline$m_{\tilde{t}_{1}}$ & 1636 & 1391 & 1760 \\
\hline$m_{\tilde{t}_{2}}$ & 2258 & 1898 & 2120 \\
\hline$m_{\tilde{b}_{1}}$ & 2255 & 1895 & 2130 \\
\hline$m_{\tilde{b}_{2}}$ & 2730 & 2282 & 2400 \\
\hline$m_{\tilde{e}_{L}}$ & 2731 & 2294 & 2680 \\
\hline$m_{\tilde{\tau}_{1}}$ & 2714 & 2255 & 2270 \\
\hline$m_{\tilde{\chi}_{1}^{ \pm}}$ & 114 & 113 & 255 \\
\hline$m_{\tilde{\chi}_{2}^{ \pm}}$ & 255 & 251 & 434 \\
\hline$m_{\widetilde{\chi}_{4}^{0}}$ & 255 & 252 & 434 \\
\hline$m_{\widetilde{\chi}_{3}^{0}}$ & 152 & 149 & 277 \\
\hline$m_{\widetilde{\chi}_{2}^{0}}$ & 136 & 134 & 267 \\
\hline$m_{\widetilde{\chi}_{1}^{0}}$ & 82 & 81 & 196 \\
\hline$m_{A}$ & 2704 & 2212 & 1720 \\
\hline$m_{H^{+}}$ & 2706 & 2214 & 1720 \\
\hline$m_{h}$ & 118 & 118 & 119 \\
\hline$\Omega_{\tilde{\chi}_{1}^{0}} h^{2}$ & 0.128 & 0.120 & 0.092 \\
\hline$B r(b \rightarrow s \gamma)$ & $3.62 \times 10^{-4}$ & $3.57 \times 10^{-4}$ & $3.42 \times 10^{-4}$ \\
\hline $\operatorname{Br}\left(B_{s} \rightarrow \mu^{+} \mu^{-}\right)$ & $3.12 \times 10^{-9}$ & $3.11 \times 10^{-9}$ & $3.02 \times 10^{-9}$ \\
\hline$\Delta a_{\mu}$ & $4.60 \times 10^{-11}$ & $1.14 \times 10^{-10}$ & $2.56 \times 10^{-10}$ \\
\hline
\end{tabular}

Table 2: mSUGRA Benchmark points A, B and $C$ (masses are in GeVs). The first five parameters define the model, while the rest are predictions.

sfermion mode has been allowed at any scale. We now comment on the differences in spectra 
between the NUSM and mSUGRA benchmark points. For reasons that have been already stated, the high scale scalar mass parameters need to be chosen differently in the two cases. Consequently, the value of $\mu$ in NUSM is larger than that in mSUGRA, simply because the mSUGRA benchmark points are within or very close to the HB/FP zones. We must note that there is no $\mathrm{HB} / \mathrm{FP}$ like effect in NUSM that would reduce $\mu$. As a result, the chargino and neutralino masses in mSUGRA benchmark points are smaller than their counterparts in NUSM.

Finally, in regard to the mass of gluino it is important to clarify the role of radiative corrections in the benchmark points of the two scenarios namely mSUGRA- $i$ and NUSM's point $i$, where $i \equiv \mathrm{A}, \mathrm{B}, \mathrm{C}$. Radiative corrections comprising of gluon-gluino and quark-squark loops may be estimated as in Eq.5 [50].

$$
\begin{aligned}
m_{\tilde{g}} & =m_{3}\left(Q^{2}\right)+\frac{3 \alpha_{s}}{4 \pi} m_{3}\left(5-3 \ln \left(\frac{m_{3}^{2}}{Q^{2}}\right)\right) \\
& -\sum_{q=u, ., t} \frac{\alpha_{s}}{4 \pi} m_{3} \operatorname{Re}\left[\hat{B}_{1}\left(m_{3}^{2}, m_{q}^{2}, m_{\tilde{q}_{1}}^{2}\right)+\hat{B}_{1}\left(m_{3}^{2}, m_{q}^{2}, m_{\tilde{q}_{2}}^{2}\right)\right] \\
& +\sum_{q=t, b} \frac{\alpha_{s}}{4 \pi} m_{q} \sin \left(2 \theta_{q}\right) \operatorname{Re}\left[B_{0}\left(m_{3}^{2}, m_{q}^{2}, m_{\tilde{q}_{1}}^{2}\right)-B_{0}\left(m_{3}^{2}, m_{q}^{2}, m_{\tilde{q}_{2}}^{2}\right)\right] .
\end{aligned}
$$

The Passarino-Veltman functions $B_{0}, \hat{B}_{1}$ and further useful details may be seen in Ref. [50]. The choice of the scale $Q$ is not unambiguous and, in general, is defined by an appropriate mass scale in the theory. In SuSpect, for example, this is set equal to the geometric average of the values of the two stop squark masses. As we can see from Table 3, this average varies widely between mSUGRA- $i$ and the corresponding NUSM's benchmark point $i$. As a result the running mass $m_{3}\left(Q^{2}\right)$ for mSUGRA- $i$ is smaller where $Q$ is higher compared to point $i$ of NUSM, where the corresponding scale is smaller because the masses of the third generation of squarks in NUSM are quite smaller ${ }^{3}$. In general, for the given benchmark points under consideration, a point mSUGRA- $i$ has a smaller running mass $m_{3}\left(Q^{2}\right)$ but has a much larger contribution from radiative corrections (vide Eq.5) compared to the corresponding point $i$ of NUSM. We note that the radiative correction amounts that arise from gluon-gluino and quark-squark loops are quite different in the two scenarios. With a heavier average squark mass, a benchmark point mSUGRA- $i$ has a much smaller contribution from quark-squark loops compared to that of NUSM point $i$. On the other hand, the logarithmic term in Eq.5 is such that for mSUGRA- $i$ the term is negative because of the fact that $m_{3}^{2}\left(Q^{2}\right)<Q^{2}$ owing to a heavier average SUSY mass scale. This leads to a large contribution from the

\footnotetext{
${ }^{3} m_{3}\left(Q^{2}\right)$ increases with a decreasing $Q$ : see for example Ref. [51].
} 


\begin{tabular}{|c||c|c|c|c|c|c|}
\hline Points & $m_{0}$ & $m_{1 / 2}$ & $\mathrm{Q}$ & $m_{3}\left(Q^{2}\right)$ & $m_{\tilde{g}}$ & Radiative Correction \\
\hline & $\mathrm{GeV}$ & $\mathrm{GeV}$ & $\mathrm{GeV}$ & $\mathrm{GeV}$ & $\mathrm{GeV}$ & \\
\hline $\mathrm{A}$ & 2050 & 270 & 357 & 633 & 709 & $12 \%$ \\
\hline mSUGRA-A & 2740 & 253 & 1917 & 548 & 740 & $35 \%$ \\
\hline B & 2000 & 255 & 329 & 602 & 674 & $12 \%$ \\
\hline mSUGRA-B & 2300 & 252 & 1619 & 551 & 725 & $32 \%$ \\
\hline C & 1250 & 540 & 903 & 1197 & 1278 & $7 \%$ \\
\hline mSUGRA-C & 2680 & 490 & 1921 & 1051 & 1273 & $21 \%$ \\
\hline
\end{tabular}

Table 3: Running mass, radiative correction in percentage and pole mass of gluino in mSUGRA and NUSM benchmark points. The scale $Q$ refers to geometric mean of stop squark mass values.

second term of the same equation for mSUGRA- $i$. This, however is not true for NUSM where one has $m_{3}^{2}\left(Q^{2}\right)>Q^{2}$ owing to a lighter average stop mass or a lighter SUSY mass scale in general. NUSM points also have significant amount of quark-squark contributions for the same reason. The final effect is such that smaller values of $m_{3}\left(Q^{2}\right)$ are overrun by radiative corrections in mSUGRA-A and mSUGRA-B leading to larger values of the pole masses $m_{\tilde{g}}$ in comparison to the values of $m_{\tilde{g}}$ for NUSM benchmark points A and B. The point mSUGRA-C and NUSM point $\mathrm{C}$ are quite competing in the above effects because of a larger associated $m_{3}$ and the values of $m_{\tilde{g}}$ are thus close to each other.

\section{Collider Signatures}

\subsection{The general strategy}

The collider signatures, and hence the optimal search strategies, of the NUSM would naturally depend on the particular point in the parameter space that nature may have chosen. Rather than attempting a general, and hence non-optimal, analysis, we choose to illustrate the various features, concentrating largely on the three representative points identified in the preceding section. To start with, we summarize, in brief, the generic simulation procedure that has been adopted here. The spectrum generated by SuSpect v2.34 as described earlier is fed into the event generator Pythia 6.4.16 [52] through a standard SLHA [53] interface for 
the simulation of $p p$ collisions with a centre-of-mass energy of $14 \mathrm{TeV}$.

We have used the CTEQ5L [54] parton distribution functions, the QCD renormalization and factorization scales both being set at the subprocess centre-of-mass energy $\sqrt{\hat{s}}$. All possible SUSY processes and decay chains consistent with conserved $R$-parity have been kept open. We have kept initial and final state radiations (ISR/FSR) on. The effect of multiple interactions has been neglected though. We, however, take hadronization into account using the fragmentation functions built into Pythia.

In Table 4, we list the total supersymmetric particle production cross-sections for each of the benchmark points. Also listed are the individual cross sections for some of the important processes, namely, $\tilde{g} \tilde{g}, \tilde{t}_{1(2)} \tilde{t}_{1(2)}^{*}$ and $\tilde{b}_{1(2)} \tilde{b}_{1(2)}^{*}$ and processes with at least one chargino or neutralino denoted by " $\tilde{\chi}_{i}^{0} / \tilde{\chi}_{1,2}^{ \pm}$". We note that, for points $\mathrm{A}$ and $\mathrm{B}$, dominant production accrues from stop pairs, while for point $\mathrm{C}$, no production process dominates overwhelmingly. The other important processes include associated stop and sbottom production along with gluinos as well as charginos and neutralinos. That the total cross-section is much smaller for point $\mathrm{C}$, compared to the other two, is easy to understand as the spectrum is relatively heavier in this case. It should be noted that while the mSUGRA and the NUSM benchmark points are quite similar as far as the gluino-pair production or the total supersymmetric particle production cross sections are concerned, they differ markedly in the dominant production modes. For the mSUGRA points, it is the lighter neutralinos and charginos that dominate overwhelmingly, whereas for the NUSM points, this rôle is usurped by stop-pairs and sbottom pairs.

\begin{tabular}{|c|c|c|c|c|c|c|c|c|}
\hline \multicolumn{4}{|c|}{ mSUGRA } & \multicolumn{5}{c|}{ NUSM } \\
\hline Point & Total & $\tilde{\chi}_{i}^{0} / \tilde{\chi}_{1,2}^{ \pm}$ & $\tilde{g} \tilde{g}$ & Point & Total & $\tilde{t}_{1} \tilde{t}_{1}^{*}+\tilde{t}_{2} \tilde{t}_{2}^{*}$ & $\tilde{b}_{1} \tilde{b}_{1}^{*}+\tilde{b}_{2} \tilde{b}_{2}^{*}$ & $\tilde{g} \tilde{g}$ \\
\hline mSUGRA-A & 11.86 & 10.67 & 1.18 & A & 12.42 & 6.77 & 1.73 & 1.28 \\
\hline mSUGRA-B & 12.49 & 11.18 & 1.25 & B & 19.92 & 11.73 & 2.79 & 1.78 \\
\hline mSUGRA-C & 0.62 & 0.59 & 0.02 & C & 1.23 & 0.09 & 0.02 & 0.07 \\
\hline
\end{tabular}

Table 4: Total supersymmetric particle production cross-sections (in pb) as well as the leading contributions for each of the NUSM and mSUGRA benchmark points.

Before we discuss the signals, it behoves us to briefly discuss the major decay modes (see Tables 5 and 6), for the structure of the cascades would determine the final state configurations. Starting with the major produce, namely the stop, for each of points A and 


\begin{tabular}{|c|c|c|c|}
\hline $\begin{array}{c}\text { Decay modes } \\
\text { (squark/gluino) }\end{array}$ & A & B & C \\
\hline \hline$\widetilde{g} \rightarrow \widetilde{b}_{1} b$ & 31.0 & 33.0 & 28.0 \\
\hline$\widetilde{g} \rightarrow \widetilde{b}_{2} b$ & 26.0 & 26.0 & 20.0 \\
\hline$\widetilde{g} \rightarrow \widetilde{t}_{1} t$ & 22.0 & 21.0 & 29.0 \\
\hline$\widetilde{g} \rightarrow \widetilde{t}_{2} t$ & 21.0 & 20.0 & 23.0 \\
\hline \hline$\widetilde{b}_{1} \rightarrow \widetilde{\chi}_{1}^{0} b$ & 8.0 & 13.0 & 7.0 \\
\hline$\widetilde{b}_{1} \rightarrow \widetilde{\chi}_{2}^{0} b$ & 42.0 & 52.0 & 24.0 \\
\hline$\widetilde{b}_{1} \rightarrow \widetilde{\chi}_{1}^{ \pm} t$ & 11.0 & 0.0 & 40.0 \\
\hline$\widetilde{b}_{1} \rightarrow \widetilde{t}_{1} W^{-}$ & 37.0 & 33.0 & 3.0 \\
\hline \hline$\widetilde{t}_{1} \rightarrow \widetilde{\chi}_{1}^{+} b$ & 100.0 & 100.0 & 33.0 \\
\hline$\widetilde{t}_{1} \rightarrow \widetilde{\chi}_{2}^{-} b$ & 0 & 0 & 21.0 \\
\hline$\widetilde{t}_{1} \rightarrow \widetilde{\chi}_{1}^{0} t$ & 0.0 & 0.0 & 23.0 \\
\hline$\widetilde{t}_{1} \rightarrow \widetilde{\chi}_{2}^{0} t$ & 0.0 & 0.0 & 13.0 \\
\hline \hline$\widetilde{\chi}_{2}^{0} \rightarrow \widetilde{\chi}_{1}^{0} q \bar{q}$ & 69.0 & 71.0 & 0.0 \\
\hline$\widetilde{\chi}_{2}^{0} \rightarrow \widetilde{\chi}_{1}^{0} l \bar{l}$ & 10.0 & 10.0 & 0.0 \\
\hline$\widetilde{\chi}_{2}^{0} \rightarrow \widetilde{\chi}_{1}^{0} \nu \bar{\nu}$ & 20.0 & 18.0 & 0.0 \\
\hline$\widetilde{\chi}_{2}^{0} \rightarrow \widetilde{\chi}_{1}^{0} h$ & 0.0 & 0.0 & 90.0 \\
\hline \hline$\widetilde{\chi}_{1}^{+} \rightarrow \widetilde{\chi}_{1}^{0} W^{+}$ & 100.0 & 100.0 & 100.0 \\
\hline
\end{tabular}

Table 5: The branching ratios(\%) of the dominant decay modes of the gluinos, squarks and lighter electroweak gauginos for NUSM for the different benchmark points.

$\mathrm{B}$, it has almost a $100 \%$ decay branching fraction to $b$ and $\tilde{\chi}_{1}^{+}$. The $\tilde{\chi}_{1}^{ \pm}$decays, in turn, into $W^{ \pm}$and the LSP again with nearly a $100 \%$ branching fraction. For point C, although $\tilde{t_{1}} \longrightarrow b \tilde{\chi}_{1}^{+}$is the dominant decay mode, the stop also has sizable branching into $t \tilde{\chi}_{1}^{0}, t \tilde{\chi}_{2}^{0}$ and $b \tilde{\chi}_{2}^{ \pm}$. As for the sbottoms, they have sizable branching fractions into both the top-chargino and the bottom-neutralino modes. The former, though slightly suppressed on account of phase space considerations, is particularly interesting in that it leads to tops in final states. With the stop and sbottom being so light in this scenario, it is obvious that the gluino decay branching fractions into stop and sbottom (accompanied by a top or a bottom, as the case may be) are significantly enhanced as compared to the typical mSUGRA case. In fact, these modes, all of comparable magnitudes, together turn out to be overwhelmingly 


\begin{tabular}{|c|c|c|c|}
\hline $\begin{array}{c}\text { Decay modes } \\
\text { (squark/gluino) }\end{array}$ & mSUGRA-A & mSUGRA-B & mSUGRA-C \\
\hline$\widetilde{g} \rightarrow \widetilde{\chi}_{1}^{ \pm} t b$ & 26.6 & 27.4 & 33.0 \\
\hline$\widetilde{g} \rightarrow \widetilde{\chi}_{2}^{ \pm} t b$ & 19.0 & 19.0 & 12.0 \\
\hline$\widetilde{g} \rightarrow \widetilde{\chi}_{i}^{0} t \bar{t}$ & 22.0 & 22.0 & 31.8 \\
\hline \hline$\widetilde{\chi}_{2}^{0} \rightarrow \widetilde{\chi}_{1}^{0} q \bar{q}$ & 33.0 & 35.4 & 54.4 \\
\hline$\widetilde{\chi}_{2}^{0} \rightarrow \widetilde{\chi}_{1}^{ \pm} u d$ & 32.4 & 32.0 & 12.0 \\
\hline$\widetilde{\chi}_{2}^{0} \rightarrow \widetilde{\chi}_{1}^{ \pm} l \nu_{l}$ & 10.0 & 15.4 & 6.0 \\
\hline \hline$\widetilde{\chi}_{1}^{+} \rightarrow \widetilde{\chi}_{1}^{0} u d$ & 66.6 & 66.6 & 66.6 \\
\hline$\widetilde{\chi}_{1}^{+} \rightarrow \widetilde{\chi}_{1}^{0} l \nu_{l}$ & 33.0 & 33.0 & 33.0 \\
\hline
\end{tabular}

Table 6: The branching ratios(\%) of the dominant decay modes of the gluinos, lighter neutralino and chargino states for mSUGRA for the different benchmark points.

dominant. This, obviously, results in an enhanced scope of having top and/or bottom quarks with a high multiplicity. This, in turn, makes it likely to have several leptons in the final state (typically from the top quark decays). This particular character of the spectrum, thus, raises hopes for $4 \ell$ (with $\ell=e, \mu$ ) final states as a viable signal of SUSY. We, nonetheless, do not limit ourselves to these alone, but consider each of the following final states:

- Opposite sign dilepton $(O S D):\left(\ell^{ \pm} \ell^{\prime \mp}\right)+(\geq 2) j e t s+\#_{T}$,

- Same sign dilepton $(S S D):\left(\ell^{ \pm} \ell^{\prime \pm}\right)+(\geq 2) j e t s+\mathbb{H}_{T}$,

- Trilepton $(3 \ell+j e t s): 3 \ell+(\geq 2)$ jets $+\not_{T}$,

- Hadronically quiet trilepton ${ }^{4}(3 \ell): 3 \ell+\not_{T}$,

- Inclusive 4-lepton $(4 \ell+X): 4 \ell+X+E_{T}$,

where $\ell$ stands for final state electrons and/or muons, $\not_{T}$ denotes missing transverse energy and $X$ denotes any associated jet(s).

Of the various final states listed above, only the hadronically quiet trileptons have their origin in electroweak processes such as $\widetilde{\chi}_{2}^{0} \widetilde{\chi}_{1}^{ \pm}$production. However, as can be seen from our event selection criteria set down in section 3.2 , strong processes which do not give rise to

\footnotetext{
${ }^{4}$ These get contributions from electroweak production of a chargino and a neutralino.
} 
hard enough jets can also be responsible for such final states. The large rate of $t \tilde{t}$ and $b \tilde{b}$ production in NUSM thus leads to relatively higher rates for hadronically quiet trileptons. On the whole, rates are never found to exceed a few percent of those with accompanying hard jets.

As is well known, in the LHC environment, even if the hard scattering process were to lead to a purely non-hadronic final state, the actual observable final state would, nonetheless, still include typically a few jets, originating from underlying events, pile up effects and ISR/FSR. In view of this, we define a hadronically quiet event to be one devoid entirely of any jet with $E_{T}{ }^{\text {jet }} \geq 100 \mathrm{GeV}$. This avoids unnecessary removal of events accompanied by relatively soft jets.

\subsection{Detection and Kinematical Requirements}

Before we mention the selection cuts, we would like to discuss the resolutions of the detectors, specifically those applicable to the ECAL, the HCAL and the muon chamber that have been incorporated in our analysis [55]. This is particularly important for reconstructing missing$E_{T}$, which is a key variable for discovering physics beyond the Standard Model.

We assume that all charged particles with $p_{T}>0.5 \mathrm{GeV}$ are detected $^{5}$ as long as they emanate within the pseudorapidity range $|\eta|<5$. For muons though, the applicable pseudorapidity range is determined by the geometry of the muon chamber to be ${ }^{6}|\eta|<2.5$. All the particles thus detected constitute the "physics objects" that are reconstructed in a collider experiments, and are further classified as

- isolated leptons;

- hadronic jets formed after identifying isolated leptons;

- unclustered energy comprised of calorimetric clusters with $p_{T}>0.5 \mathrm{GeV}$ (ATLAS) and $|\eta|<5$, that are not associated with any of the above types of high- $E_{T}$ objects

Electrons and muons with $p_{T}>10 \mathrm{GeV}$ and $|\eta|<2.5$ may be identified as isolated leptons. In order to be deemed isolated, the lepton should be sufficiently separated from

\footnotetext{
${ }^{5}$ This threshold is specific to ATLAS, while for CMS, $p_{T}>1 \mathrm{GeV}$ is applicable. Our results, though, are largely insensitive to the exact figure.

${ }^{6}$ Although it seems that muons in the range $2.5<\mid \eta<5$ would leave their footprints in the tracker, we deliberately choose to be consistent with the above criteria. Once again, the inclusion of such muons would make little quantitative difference.
} 
any other lepton in that it must satisfy $\Delta R_{\ell \ell} \geq 0.2$, where $\Delta R=\sqrt{(\Delta \eta)^{2}+(\Delta \phi)^{2}}$ is the separation in the pseudorapidity-azimuthal angle plane. Similarly, it must be far away $\left(\Delta R_{\ell j} \geq 0.4\right)$ from all putative jets with $E_{T}>20 \mathrm{GeV}$. And, finally, the total energy deposit from all hadronic activity within a cone of $\Delta R \leq 0.2$ around the the lepton axis should be $\leq 10 \mathrm{GeV}$.

Jets are formed with all the final state particles after removing the isolated leptons from the list with PYCELL, the inbuilt cluster routine in Pythia. The detector is assumed to stretch over the pseudorapidity range $|\eta| \leq 5$ and is segmented into 100 equal-sized (in $\eta$-spread) strips. Similarly, the entire $2 \pi$ azimuthal spread is again segmented into 64 equal-sized strips resulting in a $100 \times 64$ grid of cells. To register a signal, a minimum $E_{T}$ of $0.5 \mathrm{GeV}$ needs to be deposited in a cell, while the minimum $E_{T}$ for a cell to act as a jet initiator is assumed to be $2 \mathrm{GeV}$. All objects within a cone of $\Delta R=0.4$ around the jet initiator cell are considered for jet formation, and for a conglomeration to be considered a jet, it must satisfy $\sum_{\text {objects }} E_{T}>20 \mathrm{GeV}$.

Now, as has been mentioned earlier, all the other final state particles, which are not isolated leptons and are yet separated from jets by $\Delta R \geq 0.4$ are considered as unclustered objects. This includes all electromagnetic objects (muons) with $0.5 \mathrm{GeV}<E_{T}<10 \mathrm{GeV}$ and $|\eta|<5(2.5)$ as well as hadronic energy deposits with $0.5 \mathrm{GeV}<E_{T}<20 \mathrm{GeV}$ and $|\eta|<5$. Such unclustered energy deposits need to be taken into account in order to properly reconstruct any missing- $E_{T}$.

Any detector suffers from finite resolutions and collider detectors are no exception. To approximate the attendant experimental effects, we smear the energies (transverse momenta) with Gaussian functions. Nominally, the widths of the distributions have different contributions (accruing from different sources), each with a characteristic energy dependence and with these being added in quadrature. To wit (all energies are measured in units of GeV),

- electron/photon energy resolution:

$$
\frac{\sigma(E)}{E}=\frac{a}{\sqrt{E}} \oplus b \oplus \frac{c}{E}
$$

where

$$
(a, b, c)= \begin{cases}(0.030,0.005,0.2) & |\eta|<1.5 \\ (0.055,0.005,0.6) & 1.5<|\eta|<5\end{cases}
$$

- muon $p_{T}$ resolution :

$$
\frac{\sigma\left(P_{T}\right)}{P_{T}}= \begin{cases}a & |\eta|<1.5 \\ a+b \log \frac{p_{T}}{100 \mathrm{GeV}} & 1.5<|\eta|<2.5\end{cases}
$$


with

$$
(a, b)= \begin{cases}(0.008,0.037) & |\eta|<1.5 \\ (0.020,0.050) & 1.5<|\eta|<2.5\end{cases}
$$

- jet energy resolution :

$$
\frac{\sigma\left(E_{T}\right)}{E_{T}}=\frac{a}{\sqrt{E_{T}}}
$$

with $a=0.55$ being the default value used in PYCELL

- unclustered energy resolution :

$$
\sigma\left(E_{T}\right)=\alpha \sqrt{\Sigma_{i} E_{T}^{(U n c . E)_{i}}}
$$

where $\alpha \approx 0.55$. One should keep in mind here that the $x$ - and $y$-components of $E_{T}^{U n c . E}$ need to be smeared independently (with identical widths).

Once we have identified the 'physics objects' as described above, we sum vectorially the transverse components of all the momenta smeared thus to obtain the total visible transverse momentum. Clearly, the missing transverse energy is nothing but the magnitude of the visible transverse momentum, namely

$$
E_{T}=\sqrt{\left(\Sigma p_{x}\right)^{2}+\left(\Sigma p_{y}\right)^{2}}
$$

where the sum goes over all the isolated leptons, the jets as well as the unclustered energy deposits. At this stage, we are in a position to impose the selection cuts, namely

- Missing transverse energy $\mathbb{H}_{T} \geq 100 \mathrm{GeV}$,

- $p_{T}^{\ell} \geq 20 \mathrm{GeV}$ for all isolated leptons,

- $E_{T}^{\mathrm{jet}} \geq 100 \mathrm{GeV}$ and $\left|\eta_{\text {jet }}\right| \leq 2.5$,

- For the hadronically quiet trilepton events, as also for inclusive $4 \ell$ events, we reject, in addition, any event with a same flavour opposite sign lepton pair satisfying $\mid M_{Z}-$ $M_{\ell^{+} \ell^{-}} \mid \leq 10 \mathrm{GeV}$. Such events are characterised by the requirement of having no central jet with $E_{T}>100 \mathrm{GeV}$.

We have generated the corresponding SM backgrounds (with identical kinematical cuts) using Pythia. The bulk of the contribution comes from $t \bar{t}$ events. To take into account 
the next to leading order (NLO) and next to leading log resummed (NLL) corrections - not included in Pythia - we rescale the results by the appropriate $K$-factor [56] viz. 2.23. Exclusive diboson $(W W, W Z, Z Z)$ production constitutes another potential background, but it is easy to see that except for the hadronically quite trilepton channel, these contributions are very sub-dominant. Furthermore, these are reduced drastically by the cuts imposed, especially by the one on the leptonic invariant mass. Inclusive, i.e. including (multi-)jets, gauge boson production is another very serious background, but can be estimated with a high accuracy using ALPGEN [57]. The combination of a large missing $E_{T}$ along with the requirement of at least two high- $p_{T}$ leptons reduces even this to innocuous levels.

\subsection{Results}

The event rates in the various channels discussed in the preceding section would, of course, differ amongst themselves and also depend on the point in the parameter space, both on account of the differing production cross sections and branching fractions as well as the kinematical restrictions imposed. In Table 7, we tabulate the event rates in different channels obtained for the points A, B and C of the NUSM scenario as well as those for the corresponding mSUGRA ones. Also shown are the respective SM backgrounds.

For the NUSM benchmark points, the gluino decays dominantly into top-stop and bottomsbottom (see table 4). The source of leptons in the final state can thus be both the stop and the sbottom which can lead to the top and chargino in the next stage of the cascade. Of course, appropriate branching and combinatoric factors are to be used in each case. For the mSUGRA benchmark points, on the other hand, the gluino (which is the lightest strongly interacting superparticle) decays primarily into the three-body channels such as $t \bar{t} \widetilde{\chi}_{i}^{0}$ and $t b \widetilde{\chi}_{i}^{ \pm}$(see table 5). It should be remembered, however, that gluino decays mediated by light squark flavours are not entirely negligible and in fact, can account for upto one-third of the decays. This causes an effective enhancement, in the NUSM cases, of decays into the intermediate states containg top/chargino.

Another crucial difference is the splitting between the $\tilde{\chi}_{1}^{ \pm}$and $\tilde{\chi}_{1}^{0}$ states. With the splitting being large in the NUSM case, the $W^{ \pm}$from $\tilde{\chi}_{1}^{ \pm}$can be nearly on-shell (as opposed to an off-shell one in the mSUGRA case), thereby resulting in typically harder leptons. Together, these features are responsible for effectively reducing the rates for leptonic final states for mSUGRA in comparison with the NUSM benchmark points consistent with the dark matter constraints. Of course, the already mentioned difference in gluino masses has also a small role to play. 


\begin{tabular}{|c|c|c|c|c|c|}
\hline Model Points & $\sigma_{O S D}$ & $\sigma_{S S D}$ & $\sigma_{3 \ell+\text { jets }}$ & $\sigma_{3 \ell}$ & $\sigma_{4 \ell}$ \\
\hline \hline A & 103 & 24.0 & 14.9 & 3.1 & 3.1 \\
\hline mSUGRA-A & 33.7 & 15.4 & 8.1 & 0.4 & 1.3 \\
\hline B & 135 & 28.7 & 19.0 & 4.4 & 3.8 \\
\hline mSUGRA-B & 38.9 & 16.9 & 9.1 & 0.4 & 1.5 \\
\hline C & 23.9 & 7.3 & 2.9 & 0.1 & 0.3 \\
\hline mSUGRA-C & 1.8 & 0.6 & 0.4 & 0.1 & 0.1 \\
\hline \multicolumn{7}{|c|}{ SM Backgrounds } \\
\hline$t \bar{t}$ & $1.10 \times 10^{3}$ & 18.1 & 2.7 & 5.3 & 0.0 \\
\hline$Z Z, W Z, Z H, Z \gamma$ & 16.3 & 0.3 & 0.5 & 1.1 & 0.4 \\
\hline Total Bkgd & $1.12 \times 10^{3}$ & 18.4 & 3.2 & 6.4 & 0.4 \\
\hline \hline
\end{tabular}

Table 7: Event-rates (fb) after cuts for non-universal scalar mass points and corresponding mSUGRA points with same gluino mass. CTEQ5L parton distribution functions used with $\mu_{F}=\mu_{R}=\sqrt{\hat{s}}$.

Based on the above observations, the following features in the results are noted.

- For each of $O S D, 3 \ell$ and $4 \ell$ final states, the difference in the absolute rates between the NUSM points and the corresponding mSUGRA ones is remarkably large. As has been argued at the beginning of Section 3.1, this can be understood in terms of the relative lightness of the third-generation squarks in the NUSM scenario. While it might be tempting to aver that this alone would serve to distinguish NUSM from mSUGRA scenarios, a little reflection shows that just the absolute rates are not enough for this purpose and a combination of observables would be required.

- For the same sign dilepton final state (a manifestation of the Majorana nature of the gauginos), the signal to background ratio $(S / B)$ exceeds unity for both points $\mathrm{A}$ and B. For point C, while $S / B<1$, a discovery is possible with an integrated luminosity of only $10 \mathrm{fb}^{-1}$. This, though, is not surprising, for $S S D$ is well known for its efficacy in SUSY search. Note though that the $S S D$ rate cannot really distinguish between NUSM and its mSUGRA counterpart. While the case for point C may look promising, it is precisely the case where the rate (and, hence, statistical significance) is low.

- While $S / B \gtrsim 1$ for the 4-lepton final state as well, the smaller rates for this signal sig- 
nificantly reduces its potential as a discovery channel. However, this could potentially serve as a very efficient discriminator between scenarios. For example, the $S / B$ ratio is close to unity even for point C.

- The situation for the $3 \ell+$ jets is somewhat better than the $4 \ell$ one. The rates are larger while maintaining the difference between NUSM and mSUGRA. Once again, for point C, the $S / B$ ratio is close to unity, while for mSUGRA-C, it is suppressed.

- As for the $O S D$ and the hadronically-quiet $3 \ell$ final states, generically, $S / B<1$. The former though boasts of the largest event rates. For points A and B, this signal begins to stand well over the background fluctuation for an integrated luminosity of as little as $1 \mathrm{fb}^{-1}$ whereas $2 \mathrm{fb}^{-1}$ would allow a discovery claim. For the hadronically-quiet trilepton mode, the required luminosity is $\sim 10 \mathrm{fb}^{-1}$ for points A and B. Qualitatively, these two points are very similar to each other, especially as far as the superpartner masses are concerned. The main difference lies in the Higgs sector, which has not been explored here.

- For point C (and even more so for mSUGRA-C), the heaviness of the spectrum translates to lower event rates.

- It is obvious that, for an integrated luminosity of $30 \mathrm{fb}^{-1}$, many of these channels would allow us to register a $5 \sigma$ discovery claim ${ }^{7}$. In Table 8, we summarise this information for each of the channels and parameter points, both in the NUSM scenario as well as their mSUGRA counterparts.

We now discuss the profile of the dominant (though not necessarily the most backgroundfree) signal mode, namely events with opposite sign dileptons. The quest is to see if quantitative features in the same could be used to either accentuate the discovery potential or as discriminators between models and/or parameter points. In Fig.2, we display the normalized (to unity) distributions of missing transverse energy, a most crucial aspect of supersymmetry signals. As a comparison of the first two panels shows, the $\mathbb{F}_{T}$ distributions for parameter points $\mathrm{A}$ and $\mathrm{B}$ look very similar, which is but a consequence of the aforementioned similarity between the corresponding spectra. Furthermore, all of them are discernibly different from those for the corresponding mSUGRA points ${ }^{8}$. That the latter are softer can be under-

\footnotetext{
${ }^{7}$ The required luminosity is much smaller for some channels and parameter points.

${ }^{8}$ Note that we are concerned here about the shape of the curve, not the absolute magnitude, which, of course, are different (vide Table 7.)
} 


\begin{tabular}{|c|c|c|c|c|c|}
\hline Model Points & $O S D$ & $S S D$ & $3 \ell+$ jets & $3 \ell$ & $4 \ell$ \\
\hline \hline A & $\sqrt{ }$ & $\sqrt{ }$ & $\sqrt{ }$ & $\sqrt{ }$ & $\sqrt{ }$ \\
\hline mSUGRA-A & $\sqrt{ }$ & $\sqrt{ }$ & $\sqrt{ }$ & $\times$ & $\sqrt{ }$ \\
\hline B & $\sqrt{ }$ & $\sqrt{ }$ & $\sqrt{ }$ & $\sqrt{ }$ & $\sqrt{ }$ \\
\hline mSUGRA-B & $\sqrt{ }$ & $\sqrt{ }$ & $\sqrt{ }$ & $\times$ & $\sqrt{ }$ \\
\hline C & $\sqrt{ }$ & $\sqrt{ }$ & $\sqrt{ }$ & $\times$ & $\times$ \\
\hline mSUGRA-C & $\times$ & $\times$ & $\times$ & $\times$ & $\times$ \\
\hline \hline
\end{tabular}

Table 8: $5 \sigma$ visibility of various signals for an integrated luminosity of $30 \mathrm{fb}^{-1} . A \sqrt{ }$ indicates a positive conclusion while $a \times$ indicates a negative one.

stood by realizing that the main production channel for the mSUGRA spectrum is $p p \rightarrow \tilde{g} \tilde{g}$ and that, unlike in the NUSM case, the gluino undergoes a three body decay, resulting in relatively less momentum imparted to the LSP. Note also that the the dominant $(t \bar{t})$ background - as displayed in the first panel - is almost as soft as the mSUGRA signals, and thus a hardening of the $E_{T}$ cut would have considerably improved the $S / B$ ratio for the NUSM cases, while worsening it for the mSUGRA ones. And, finally, for point C, the difference is even more stark. However, with the size of the signal being small in this case, it is not immediately apparent whether this could be used to any advantage.

Another kinematical variable often used advantageously in searches for new physics involving $E_{T}$ is the "effective mass" defined to be the scalar sum of the transverse momenta of the isolated leptons and jets and the missing transverse energy, viz.

$$
m_{\mathrm{eff}} \equiv \sum\left(p_{T}\right)_{\text {iso } \ell}+\sum\left(p_{T}\right)_{j e t s}+\#_{T} .
$$

In Fig.3, we display the corresponding distributions, again for both the NUSM points and their mSUGRA counterparts. As the first panel shows, as far as point A is concerned, there is little to choose between this distribution and that for the corresponding mSUGRA point. Similar is the case for point B (second panel). Note, furthermore, that the peak in either case is at a fairly large value of $m_{\text {eff }}$. While this, at first sight, might seem contradictory to the oft-repeated claim that this distribution should peak roughly at twice the mass of the dominant particle being produced, the reason for this discrepancy is easy to appreciate. First and foremost, with the strong demands made on the transverse momenta of the two leading jets, the contribution from stop-pair production reduces drastically. This is understandable since the relatively small difference between the stop mass and those of the lighter chargino 

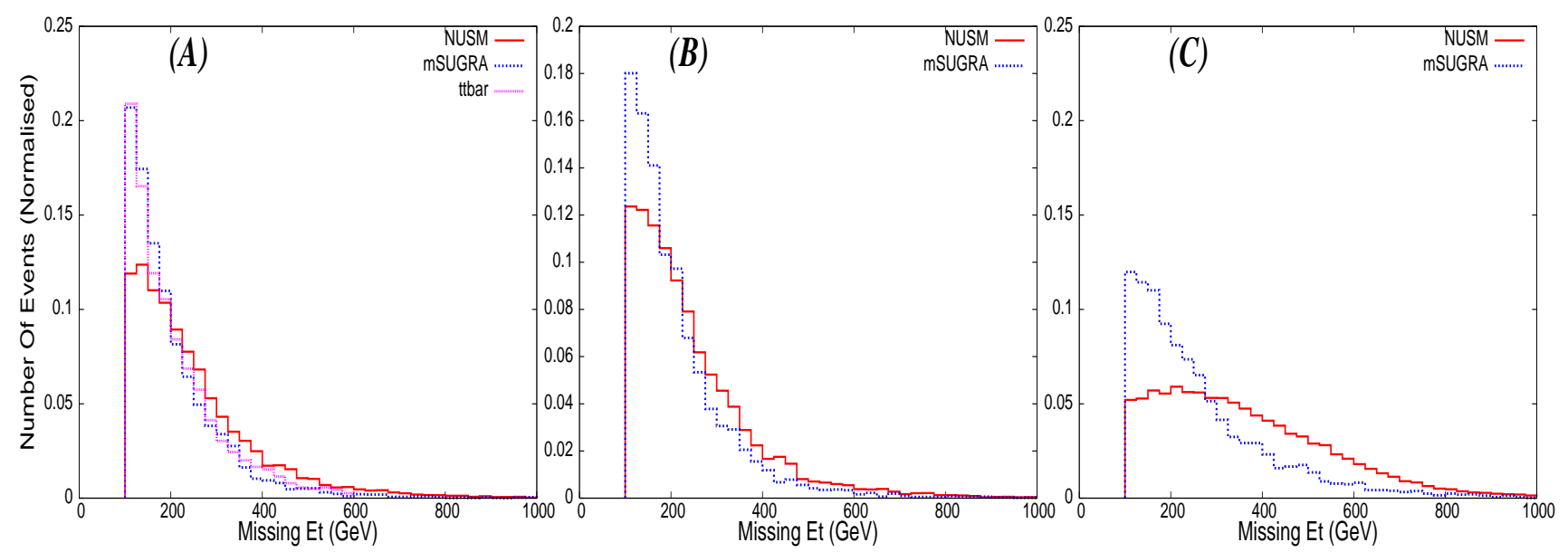

Figure 2: Missing transverse energy distribution (normalized to unit area) for opposite sign dilepton (OSD) events. The eponymous panels refer to the respective representative points in the parameter space. Also shown are the analogous distributions for the corresponding mSUGRA points. The first panel also shows the distribution accruing from the overwhelmingly dominant SM background, namely $t \bar{t}$ production.

implies that the $b$ from stop decay tends to be softer. With the stop-pair contribution thus being effectively decimated ${ }^{9}$, this also offers hints as to why the NUSM and mSUGRA distributions look so similar. And, with the gluinos themselves being produced with a considerable transverse momentum, it is easy to understand why the distribution peaks at a high value of $m_{\mathrm{eff}}$.

For point $\mathrm{C}$ though, a remarkable difference with the mSUGRA counterpart is immediately apparent. This can be traced to the fact that the charginos are considerably softer for mSUGRA-C as compared to the point C. This allows for the second, subdominant, hump slightly above twice the mass of the chargino. While this could, in principle, be used to discriminate between the two scenarios, unfortunately the smaller rates tend to make the task a difficult one. And, finally, note that the distribution for the (dominant) $t \bar{t}$ background is considerably softer than those for any of the six supersymmetric parameter points discussed

\footnotetext{
${ }^{9}$ It might seem paradoxical that we are altogether sacrificing the signal from the light stop, a cornerstone of this scenario. However, including the stop contribution would require softening the $p_{T}$ requirements, a process fraught with danger in the context of the LHC. In the absence of a full-scale simulation including multiple scattering and underlying events, we deliberately desist from this.
} 

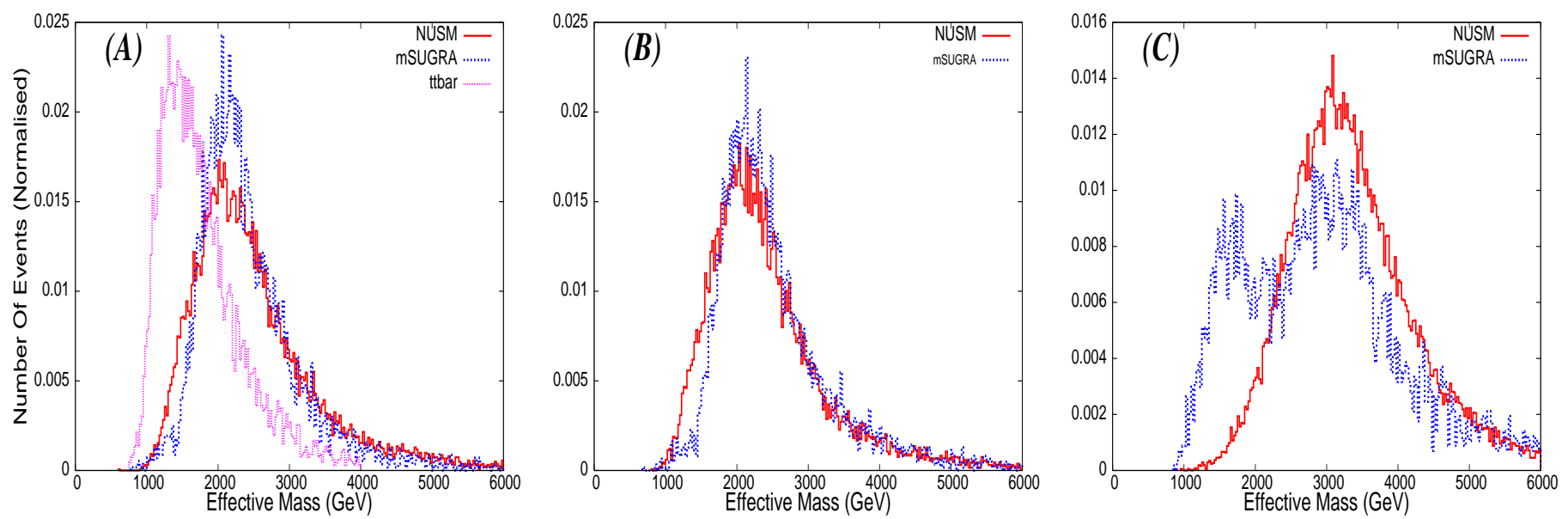

Figure 3: As in Fig.2, but for the effective mass instead.

here. Thus, imposing a requirement such as $m_{\mathrm{eff}} \gtrsim 2 \mathrm{TeV}$ would have significantly improved the $S / B$ ratio for the $O S D$ signal. This, though, would have eliminated the secondary hump for the mSUGRA-C case.

Having discussed the prospects of refining and/or using the kinematical variables in the $O S D$ sample towards discriminating between scenarios, we now consider a set of observables, namely the ratios of events seen in various channels. As is well known, there is a great advantage to the use of such variables in that it almost entirely eliminates some systematic uncertainties such as that in the luminosity and drastically reduces others such as those corresponding to the choice of the parton distributions, the choice of renormalization and fragmentation scales etc. In Fig.4, we present the ratio of the subordinate channels with the dominant $(O S D)$ channel for each of the parameter points.

At this stage, we can easily formulate the means of discriminating between a NUSM point and the corresponding mSUGRA one, namely

* For parameter points (such as A and B) with a relatively smaller $m_{1 / 2}$ but large $m_{0}$ (i.e., when the gluino is considerably heavier than the stop/sbottom but sufficiently lighter than the first two generation squarks and sleptons), the NUSM scenario would typically result in a smaller proportion of same sign dilepton events, as is clear from Fig.4(a), when compared to the $O S D$ rates. This can be attributed to the fact that the $O S D$-rate increases significantly for the gluinos decaying through stop-top (with, consequently, $O S D$ being possible even from the decay of a single gluino, irrespective 

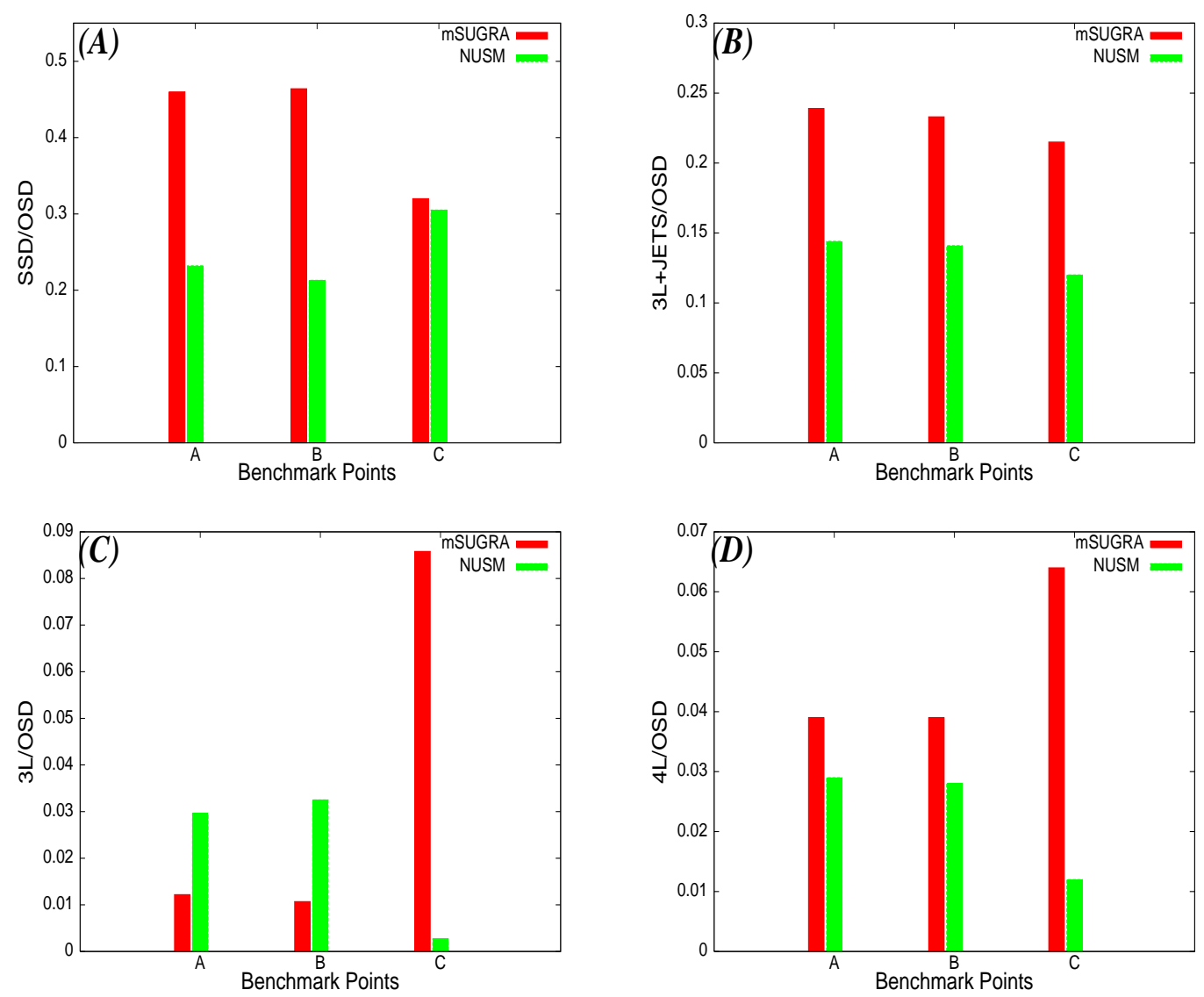

Figure 4: Event ratios with respect to opposite-sign dilepton(OSD) events for NUSM and mSUGRA cases at the points A,B,C mentioned in the text. Colour Code: Red: mSUGRA, Green: NUSM scenario.

of how the other one decays), whereas the $S S D$ relies on the good old fact of the gluino being a Majorana spinor, with only a slight increment to the leptonic branching fraction due to the decays through third generation.

* The mSUGRA-A and mSUGRA-B points have sufficiently small values of $\mu$ and these points indeed fall in the $\mathrm{HB} / \mathrm{FP}$ region. This implies that there is more of Higgsino in the lighter chargino $\left(\tilde{\chi}_{1}^{ \pm}\right)$and the second lightest neutralino $\left(\tilde{\chi}_{2}^{0}\right)$. Therefore, the leptonic signals are weakened compared to what we predict in NUSM.

* Again, for points with a small $m_{1 / 2}$ but large $m_{0}$ (such as A and B), the rates (absolute and relative) for the hadronically quiet trilepton mode are markedly higher for the 
NUSM case. This can be attributed to the aforementioned feature of the NUSM spectrum which renders it easier to have large- $p_{T}$ isolated leptons. The turnaround for point $\mathrm{C}$ vs mSUGRA-C is a consequence of the lightness of the charginos in the latter allowing a large contribution through the production of the charginos and the second-lightest neutralino.

* Overall, it is self-evident that a combination of these ratios would serve to easily distinguish between the two scenarios.

* And, finally, the relative lightness of the stop and the sbottom (and the consequent fact of the gluinos decaying through these), renders the NUSM signal $b$-rich. Invoking $b$-tagging (which we had not done in the results presented so far) would thus present us with a very useful discriminator. With this in view, we perform a study in $O S D$ channel associated with two or more partonic $b$-jets $(\geq 2 b+O S D)$. We assume a $b$ tagging efficiency [58] of $\epsilon_{b}=0.5$ for $p_{T}>40 \mathrm{GeV}$ and $|\eta|<2.5$. The $O S D$ event selection criteria remain the same. As expected, we see a clear distinction between the NUSM and the corresponding mSUGRA ones in the absolute event rates as shown in Table 9. With the NUSM sample being particularly rich in $b$ 's, the suppression in rates as compared to those in Fig.7 is understandably less severe than $\epsilon_{b}^{2}$. This, of course, does not apply as well to point $\mathrm{C}$ and far less to the MSSM cases, resulting in a large suppression of the latter.

\begin{tabular}{|c|c|c|c|c|c|c|c|}
\hline Model Points & A & mSUGRA-A & B & mSUGRA-B & C & mSUGRA-C & $t \bar{t}$ \\
\hline \hline$\geq 2 b+O S D$ & 36.6 & 6.3 & 46.4 & 10.2 & 6.7 & 0.5 & 148.7 \\
\hline \hline
\end{tabular}

Table 9: Event rates (fb) at different benchmark points and for the $t \bar{t}$ background for a final state $\geq 2 b+O S D$.

\section{Summary and Conclusions}

We have studied a case of nonuniversal scalar masses, wherein the first two families of squarks as well as sleptons of all generations are much heavier than the third family of squarks and the Higgs scalars. The universality of gaugino masses has been adhered to. We confine ourselves only to that region of the parameter space where one achieves a relic 
density consistent with the WMAP data. LSP annihilation is efficiently mediated by the pseudoscalar Higgs, with the 'funnel region' being significantly extended toward small values of $\tan \beta$ when compared to mSUGRA. Having ensured that the region of SUSY parameter space thus isolated is consistent with all constraints from FCNC and CP-violation, we have proceeded to investigate the signals of this scenario at the LHC.

Although stop-pair production is the dominant SUSY process in such scenarios, the stringent cuts that we choose to impose results in stop-cascades being suppressed. Rather, the dominant contribution to the signal rates turns out to be gluino pair-production. The relatively large multiplicity of top quarks produced in the cascades results in enhanced rates for two, three and four-lepton final states, together with missing- $E_{T}$ and hard jets. In particular, the usefulness of four-lepton final states is highlighted through this analysis.

Based on the study of a few benchmark points (corresponding to different gluino masses and $\tan \beta$ ), we find that, using the criteria chosen by us, it is possible to probe the above scenario with an integrated luminosity of $30 \mathrm{fb}^{-1}$ for gluino masses up to about $1.2 \mathrm{TeV}$. (Indeed, for certain significant parts of the parameter space, even $2 \mathrm{fb}^{-1}$ would be enough.) The reach can be potentially extended further once more luminosity accrues. We also demonstrate that it is possible to distinguish this scenario from an mSUGRA-one tuned at the same gluino mass and satisfying the WMAP constraints. The usefulness of the ratios of events in various channels is clearly elicited from our study. Moreover, such distinction is facilitated by the effective mass distribution of events for gluino masses on the higher side, i.e. above a TeV. Thus, we succeed in illustrating that a multichannel analysis is not only able to probe such nonuniversal SUSY scenarios satisfying the relic density constraints, but can also highlight notable differences with a simple-minded model based on universal SUGRA.

\section{Acknowledgments}

UC, DC and BM thank the organisers of the workshop "TeV Scale Physics and Dark Matter", held in NORDITA, Stockholm in 2008 for the kind hospitality received. The work was initiated in this workshop. The work of SB and BM was partially supported by funding available from the Department of Atomic Energy, Government of India for the Regional Centre for Accelerator-based Particle Physics, Harish-Chandra Research Institute. SB thanks Sanjoy Biswas for useful discussions. DC acknowledges support from the Department of Science and Technology, India under project number SR/S2/RFHEP-05/2006. 


\section{References}

[1] For reviews on Supersymmetry, see, e.g., H. P. Nilles, Phys. Rep. 1, 110 ( 1984); H. E. Haber and G. Kane, Phys. Rep. 117, 75 ( 1985) ; J. Wess and J. Bagger, Supersymmetry and Supergravity, 2nd ed., (Princeton, 1991); M. Drees, R. Godbole and P. Roy, Hackensack, USA: World Scientific (2004) 555 p; P. Binetruy, Oxford, UK: Oxford Univ. Pr. (2006) 520 p.

[2] S. P. Martin, arXiv:hep-ph/9709356; D. J. H. Chung, L. L. Everett, G. L. Kane, S. F. King, J. D. Lykken and L. T. Wang, Phys. Rept. 407, 1 (2005).

[3] A. H. Chamseddine, R. Arnowitt and P. Nath, Phys. Rev. Lett. 49, 970 (1982); R. Barbieri, S. Ferrara and C. A. Savoy, Phys. Lett. B 119, 343 (1982); L. J. Hall, J. Lykken and S. Weinberg, Phys. Rev. D 27, 2359 (1983); P. Nath, R. Arnowitt and A. H. Chamseddine, Nucl. Phys. B 227, 121 (1983); N. Ohta, Prog. Theor. Phys. 70, 542 (1983); For reviews see [1] and P. Nath, R. Arnowitt and A.H. Chamseddine, Applied $N=1$ Supergravity (World Scientific, Singapore, 1984).

[4] N. Polonsky and A. Pomarol, Phys. Rev. D 51, 6532 (1995); R. Barbieri, L. J. Hall and A. Strumia, Nucl. Phys. B 445, 219 (1995); R. L. Arnowitt and P. Nath, Phys. Rev. D 56, 2833 (1997).

[5] U. Chattopadhyay and D. Das, Phys. Rev. D 79, 035007 (2009).

[6] V. Berezinsky, A. Bottino, J. Ellis, N. Forrengo, G. Mignola, and S. Scopel, Astropart. Phys.5:1-26(1996).

[7] P. Nath and R. Arnowitt, Phys. Rev. D 56, 2820 (1997); D. Feldman, Z. Liu and P. Nath, JHEP 0804, 054 (2008); C. F. Berger, J. S. Gainer, J. L. Hewett and T. G. Rizzo, JHEP 0902, 023 (2009).

[8] D. G. Cerdeno and C. Munoz, JHEP 0410, 015 (2004).

[9] J. R. Ellis, T. Falk, K. A. Olive and Y. Santoso, Nucl. Phys. B 652, 259 (2003); J. R. Ellis, K. A. Olive, Y. Santoso and V. C. Spanos, Phys. Lett. B 603, 51 (2004); A. De Roeck, J. R. Ellis, F. Gianotti, F. Moortgat, K. A. Olive and L. Pape, Eur. Phys. J. C 49, 1041 (2007); J. R. Ellis, K. A. Olive and P. Sandick, Phys. Rev. D 78, $075012(2008)$. 
[10] H. Baer, A. Mustafayev, E. K. Park, S. Profumo and X. Tata, JHEP 0604, 041 (2006); H. Baer, A. Mustafayev, E. K. Park and X. Tata, JHEP 0805, 058 (2008).

[11] H. Baer, A. Mustafayev, S. Profumo, A. Belyaev and X. Tata, JHEP 0507, 065 (2005); Phys. Rev. D 71, 095008 (2005).

[12] M. Drees, Phys. Lett. B 181 (1986) 279; J. S. Hagelin and S. Kelley, Nucl. Phys. B 342 (1990) 95.

[13] Y. Kawamura, H. Murayama and M. Yamaguchi, Phys. Lett. B 324 (1994) 52; Phys. Rev. D 51 (1995) 1337.

[14] A. Datta, A. Datta and M. K. Parida, Phys. Lett. B 431, 347 (1998); A. Datta, A. Datta, M. Drees and D. P. Roy, Phys. Rev. D 61, 055003 (2000).

[15] S. Bhattacharya, A. Datta and B. Mukhopadhyaya, Phys. Rev. D 78, 035011 (2008).

[16] A. Corsetti and P. Nath, Phys. Rev. D 64, 125010 (2001); U. Chattopadhyay, A. Corsetti and P. Nath, Phys. Rev. D 66, 035003 (2002); U. Chattopadhyay and P. Nath, Phys. Rev. D 65, 075009 (2002); U. Chattopadhyay and D. P. Roy, Phys. Rev. D 68, 033010 (2003); G. Belanger, F. Boudjema, A. Cottrant, A. Pukhov and A. Semenov, Nucl. Phys. B 706, 411 (2005); K. Huitu, J. Laamanen, P. N. Pandita and S. Roy, Phys. Rev. D 72, 055013 (2005); U. Chattopadhyay, D. Choudhury and D. Das, Phys. Rev. D 72, 095015 (2005); S. F. King, J. P. Roberts and D. P. Roy, JHEP 0710, 106 (2007); K. Huitu, R. Kinnunen, J. Laamanen, S. Lehti, S. Roy and T. Salminen, Eur. Phys. J. C 58, 591 (2008); U. Chattopadhyay, D. Das and D. P. Roy, Phys. Rev. D 79, 095013 (2009); S. P. Martin, arXiv:0903.3568 [hep-ph]; D. Feldman, Z. Liu and P. Nath, arXiv:0905.1148 [hep-ph].

[17] S. Bhattacharya, A. Datta and B. Mukhopadhyaya, JHEP 0710, 080 (2007); Phys. Rev. D 78, 115018 (2008); S. Bhattacharya and J. Chakrabortty, arXiv:0903.4196 [hep-ph].

[18] S. K. Soni and H. A. Weldon, Phys. Lett. B126, 215(1983); V. S. Kaplunovsky and J. Louis, Phys. Lett. B306, 268(1993).

[19] M. Dine, R. Leigh, and A. Kagan, Phys. Rev. D48, 4269 (1993); P. Pouliot and N. Seiberg, Phys. Lett. B318, 169 (1993); Y. Nir and N. Seiberg, Phys. Lett. B309, 
337 (1993). S. Dimopoulos and G.F. Giudice, Phys. Lett. B357, 573 (1995); D. Choudhury, F. Eberlein, A. Konig, J. Louis and S. Pokorski, Phys. Lett. B 342, 180 (1995); A. Cohen, D.B. Kaplan, and A.E. Nelson, Phys. Lett. B388, 588 (1996); A. Pomarol and D. Tommasini, Nucl. Phys. B 466, 3 (1996); M. Misiak, S. Pokorski and J. Rosiek, Adv. Ser. Direct. High Energy Phys. 15, 795 (1998); P. H. Chankowski, K. Kowalska, S. Lavignac and S. Pokorski, arXiv:hep-ph/0507133.

[20] F. Gabbiani, E. Gabrielli, A. Masiero and L. Silvestrini, Nucl. Phys. B 477, 321 (1996); N. Arkani-Hamed and H. Murayama, Phys. Rev. D 56, 6733 (1997).

[21] R. Barbieri and G.F. Giudice, Nucl. Phys. B306,63(1988); P. Ciafaloni and A. Strumia, Nucl. Phys. B494, 41(1997).

[22] V. D. Barger, C. Kao and R. J. Zhang, Phys. Lett. B 483, 184 (2000).

[23] J. L. Evans, D. E. Morrissey and J. D. Wells, Phys. Rev. D 75, 055017 (2007).

[24] W. Buchmuller, J. Kersten and K. Schmidt-Hoberg, JHEP 0602, 069 (2006); W. Buchmuller, L. Covi, J. Kersten and K. Schmidt-Hoberg, JCAP 0611, 007 (2006).

[25] J. Bagger, J. L. Feng and N. Polonsky, Nucl. Phys. B 563, 3 (1999); . K. Agashe and M. Graesser, Phys. Rev. D 59, 015007 (1999); J. A. Bagger, J. L. Feng, N. Polonsky and R. J. Zhang, Phys. Lett. B 473, 264 (2000); Phys. Lett. B 475, 289 (2000); H. Baer, C. Balazs, P. Mercadante, X. Tata and Y. Wang, Phys. Rev. D 63, 015011 (2001).

[26] E. Komatsu et al. [WMAP Collaboration], arXiv:0803.0547 [astro-ph].

[27] H. Baer, C. h. Chen, F. Paige and X. Tata, Phys. Rev. D 53, 6241 (1996); H. Baer, V. Barger, A. Lessa and X. Tata, arXiv:0907.1922 [hep-ph].

[28] U. Chattopadhyay, D. Das, A. Datta and S. Poddar, Phys. Rev. D 76, 055008 (2007); N. Bhattacharyya, A. Datta and S. Poddar, Phys. Rev. D 78, 075030 (2008).

[29] K. L. Chan, U. Chattopadhyay and P. Nath, Phys. Rev. D 58, 096004 (1998); U. Chattopadhyay, A. Corsetti and P. Nath, Phys. Rev. D 68, 035005 (2003); D. Feldman, Z. Liu and P. Nath, Phys. Rev. D 78, 083523 (2008).

See also: 1st article of Ref. [34]. 
[30] J. L. Feng, K. T. Matchev and T. Moroi, Phys. Rev. D 61, 075005 (2000); J. L. Feng, K. T. Matchev and T. Moroi, Phys. Rev. Lett. 84, 2322 (2000); J. L. Feng, K. T. Matchev and F. Wilczek, Phys. Lett. B 482, 388 (2000); U. Chattopadhyay, A. Datta, A. Datta, A. Datta and D. P. Roy, Phys. Lett. B 493, 127 (2000); U. Chattopadhyay, T. Ibrahim and D. P. Roy, Phys. Rev. D 64, 013004 (2001); U. Chattopadhyay, T. Ibrahim and D. P. Roy, Phys. Rev. D 64, 013004 (2001); J. L. Feng and F. Wilczek, Phys. Lett. B 631, 170 (2005); S. P. Das, A. Datta, M. Guchait, M. Maity and S. Mukherjee, Eur. Phys. J. C 54, 645 (2008).

[31] M. Drees and M. Nojiri, Phys. Rev. D47, 376 (1993).

[32] R. Arnowitt and P. Nath, Phys. Rev. Lett. 70, 3696 (1993); H. Baer and M. Brhlik, Phys. Rev. D53, 597 (1996), Phys. Rev. D 57, 567 (1998); H. Baer, M. Brhlik, M. Diaz, J. Ferrandis, P. Mercadante, Phys. Rev. D63, 015007 (2001); J. R. Ellis, T. Falk, G. Ganis, K. A. Olive and M. Srednicki, Phys. Lett. B 510, 236 (2001); A. B. Lahanas and V. C. Spanos, Eur. Phys. J. C 23, 185 (2002); A. Djouadi, M. Drees and J. Kneur, Phys. Lett. B 62460 (2005).

[33] G. Jungman, M. Kamionkowski and K.Greist, Phys. Rep. 267,195(1995).

[34] A. B. Lahanas, N. E. Mavromatos and D. V. Nanopoulos, Int. J. Mod. Phys. D 12, 1529 (2003); C. Munoz, Int. J. Mod. Phys. A 19, 3093 (2004); M. Drees, Plenary talk at 11th International Symposium on Particles, Strings and Cosmology (PASCOS 2005), Gyeongju, Korea, 30 May - 4 Jun 2005 (published in AIP Conf.Proc., 805, 48-54, (2006).

[35] A. Djouadi, J. L. Kneur and G. Moultaka, [arXiv:hep-ph/0211331].

[36] G. Belanger, F. Boudjema, A. Pukhov and A. Semenov, Comput. Phys. Commun. 176, 367 (2007).

[37] R. Barate et al. [LEP Working Group for Higgs boson searches], Phys. Lett. B 565, 61 (2003).

[38] F. Margaroli [CDF and D0 Collaborations], Nuovo Cim. 123B, 763 (2008); T. Aaltonen et al. [CDF Collaboration], arXiv:0907.0810 [hep-ex]; arXiv:0906.5613 [hep-ex]; arXiv:0905.3155 [hep-ex]. 
[39] G. Degrassi, S. Heinemeyer, W. Hollik, P. Slavich and G. Weiglein, Eur. Phys. J. C 28, 133 (2003); B. C. Allanach, A. Djouadi, J. L. Kneur, W. Porod and P. Slavich, JHEP 0409, 044 (2004); S. Heinemeyer, hep-ph/0408340; Int. J. Mod. Phys. A 21, 2659 (2006); S. Heinemeyer, W. Hollik and G. Weiglein, Phys. Rep. 425, 265 (2006).

[40] A. L. Kagan and M. Neubert, Eur. Phys. J. C 7, 5 (1999); K. G. Chetyrkin, M. Misiak and M. Munz, Phys. Lett. B 400, 206 (1997) [Erratum-ibid. B 425, 414 (1998)]; P. Gambino and M. Misiak, Nucl. Phys. B 611, 338 (2001).

[41] M. Ciuchini, G. Degrassi, P. Gambino and G. F. Giudice, Nucl. Phys. B 527, 21 (1998); M. Ciuchini, G. Degrassi, P. Gambino and G. F. Giudice, Nucl. Phys. B 534, 3 (1998); G. Degrassi, P. Gambino and G. F. Giudice, JHEP 0012, 009 (2000).

[42] S. Chen et al. [CLEO Collaboration], Phys. Rev. Lett. 87, 251807 (2001); B. Aubert et al. [BaBar Collaboration], [arXiv:hep-ex/0207076]; P. Koppenburg et al. [Belle Collaboration], Phys. Rev. Lett. 93, 061803 (2004).

[43] A. Djouadi, M. Drees and J. L. Kneur, JHEP 0603, 033 (2006).

[44] K. i. Okumura and L. Roszkowski, Phys. Rev. Lett. 92, 161801 (2004); J. Foster, K. i. Okumura and L. Roszkowski, JHEP 0603, 044 (2006).

[45] S. R. Choudhury and N. Gaur, Phys. Lett. B 451, 86 (1999); K. S. Babu and C. Kolda, Phys. Rev. Lett. 84, 228 (2000); A. Dedes, H. K. Dreiner, U. Nierste, and P. Richardson, Phys. Rev. Lett. 87, 251804 (2001); R. Arnowitt, B. Dutta, T. Kamon and M. Tanaka, Phys. Lett. B 538 (2002) 121; J. K. Mizukoshi, X. Tata and Y. Wang, Phys. Rev. D 66, 115003 (2002); S. Baek, P. Ko, and W. Y. Song, JHEP 0303, 054 (2003); T. Ibrahim and P. Nath, Phys. Rev. D 67, 016005 (2003); J.R. Ellis, K.A. Olive and V.C. Spanos, Phys. Lett. B 624, 47 (2005).

[46] T. Aaltonen et al. [CDF Collaboration], Phys. Rev. Lett. 100, 101802 (2008).

[47] C. Bobeth, T. Ewerth, F. Kruger and J. Urban, Phys. Rev. D 64, 074014 (2001).

[48] H.E. Haber and Y. Nir, Phys. Lett. B306 327 (1993); H.E. Haber, hep-ph/9505240; A. Dobado, M.J. Herrero and S. Penaranda, Eur. Phys. J. C17 487 (2000); J.F. Gunion and H.E. Haber, Phys. Rev. D67 075019 (2003).

[49] E. Boos, A. Djouadi, M. Muhlleitner and A. Vologdin, Phys. Rev. D66 055004 (2002); E. Boos, A. Djouadi and A. Nikitenko, Phys. Lett. B578, 384 (2004); E. Boos, V. 
Bunichev, A. Djouadi and H.J. Schreiber, Phys. Lett. B622 311 (2005); A. Djouadi and Y. Mambrini, JHEP 0612, 001 (2006).

[50] D. Pierce and A. Papadopoulos, Nucl. Phys. B 430, 278 (1994); D. M. Pierce, J. A. Bagger, K. T. Matchev and R. j. Zhang, Nucl. Phys. B 491, 3 (1997).

[51] L. E. Ibanez and C. Lopez, Nucl. Phys. B 233, 511 (1984).

[52] T. Sjostrand, S. Mrenna and P. Skands, JHEP 0605, 026 (2006).

[53] P. Skands et al., JHEP 0407, 036 (2004).

[54] H. L. Lai et al. [CTEQ Collaboration], Eur. Phys. J. C 12, 375 (2000).

[55] Private communication with Bruce Melado; S. Biswas and B. Mukhopadhyaya, arXiv:0902.4349 [hep-ph].

[56] M. Cacciari, S. Frixione, M. L. Mangano, P. Nason and G. Ridolfi, JHEP 0809 (2008) 127.

[57] M. L. Mangano, M. Moretti, F. Piccinini, R. Pittau and A. D. Polosa, JHEP 0307 (2003) 001.

[58] ATLAS detector and physics performance. Technical design report. Vol. 2, CERNLHCC-99-15, ATLAS-TDR-15. 


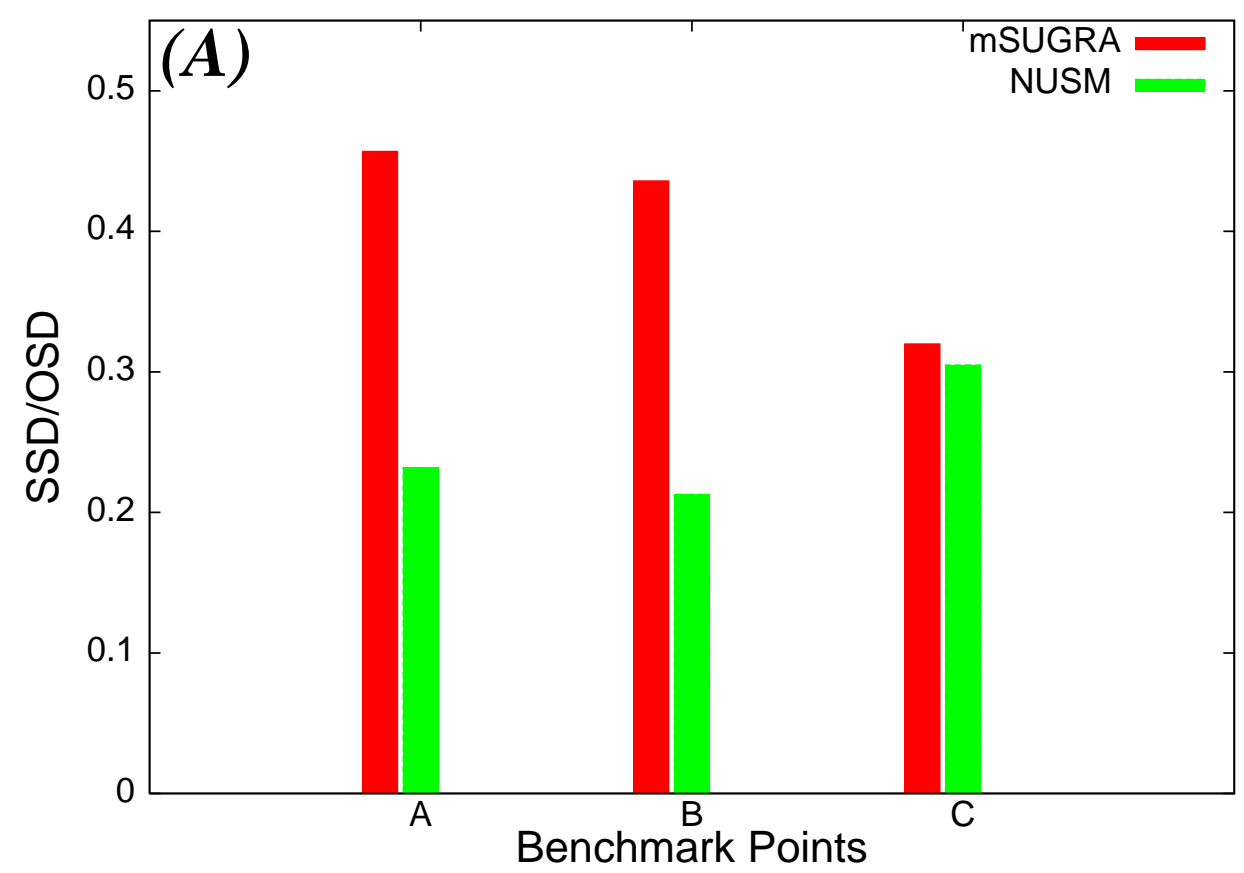

